\title{
A systematic analysis of Nrf2 pathway activation dynamics during repeated xenobiotic exposure
}

\author{
Luc J. M. Bischoff ${ }^{1}$. Isoude A. Kuijper ${ }^{1}$. Johannes P. Schimming ${ }^{1}$. Liesanne Wolters ${ }^{1}$ - Bas ter Braak ${ }^{1}$. \\ Jan P. Langenberg ${ }^{2} \cdot$ Daan Noort ${ }^{2} \cdot$ Joost B. Beltman ${ }^{1} \cdot$ Bob van de Water $^{1}$
}

Received: 3 July 2018 / Accepted: 8 November 2018 / Published online: 20 November 2018

(c) The Author(s) 2018

\begin{abstract}
Oxidative stress leads to the activation of the Nuclear factor-erythroid-2-related factor 2 (Nrf2) pathway. While most studies have focused on the activation of the Nrf2 pathway after single chemical treatment, little is known about the dynamic regulation of the Nrf2 pathway in the context of repeated exposure scenarios. Here we employed single cell live imaging to quantitatively monitor the dynamics of the Nrf2 pathway during repeated exposure, making advantage of two HepG2 fluorescent protein reporter cell lines, expressing GFP tagged Nrf2 or sulfiredoxin 1 (Srxn1), a direct downstream target of Nrf2. High throughput live confocal imaging was used to measure the temporal dynamics of these two components of the Nrf2 pathway after repeated exposure to an extensive concentration range of diethyl maleate (DEM) and tert-butylhydroquinone (tBHQ). Single treatment with DEM or tBHQ induced Nrf2 and Srxn1 over time in a concentration-dependent manner. The Nrf2 response to a second treatment was lower than the response to the first exposure with the same concentration, indicating that the response is adaptive. Moreover, a limited fraction of individual cells committed themselves into the Nrf2 response during the second treatment. Despite the suppression of the Nrf2 pathway, the second treatment resulted in a three-fold higher Srxn1-GFP response compared to the first treatment, with all cells participating in the response. While after the first treatment Srxn1-GFP response was linearly related to Nrf2-GFP nuclear translocation, such a linear relationship was less clear for the second exposure. siRNA-mediated knockdown demonstrated that the second response is dependent on the activity of Nrf2. Several other, clinically relevant, compounds (i.e., sulphorophane, nitrofurantoin and CDDO-Me) also enhanced the induction of Srxn1-GFP upon two consecutive repeated exposure. Together the data indicate that adaptation towards prooxidants lowers the Nrf2 activation capacity, but simultaneously primes cells for the enhancement of an antioxidant response which depends on factors other than just Nrf2. These data provide further insight in the overall dynamics of stress pathway activation after repeated exposure and underscore the complexity of responses that may govern repeated dose toxicity.
\end{abstract}

Keywords Nrf2 $\cdot$ Srxn1 $\cdot$ Repeated exposure $\cdot$ HepG $2 \cdot$ Oxidative stress $\cdot$ Soft electrophiles

Luc J. M. Bischoff and Isoude A. Kuijper contributed equally to the work.

Electronic supplementary material The online version of this article (https://doi.org/10.1007/s00204-018-2353-2) contains supplementary material, which is available to authorized users.

Bob van de Water

b.water@lacdr.leidenuniv.nl

1 Division of Drug Discovery and Safety, Leiden Academic Centre for Drug Research, Leiden University, Einsteinweg 55, 2333 CC Leiden, The Netherlands

2 Department of CBRN Protection, TNO Defence, Safety and Security, Rijswijk, The Netherlands

\section{Introduction}

Chemical exposure leads to the activation of various cellular stress response pathways (Jennings et al. 2013; Souza et al. 2017). These cellular stress response pathways are typically activated to initiate repair of cell injury and/or to adapt cells to possible subsequent harmful situations (Baird and Dinkova-Kostova 2011; Kensler et al. 2007). Alternatively, cell injury may initiate the activation of cell death programs to switch on self-demise of cells (Danial and Korsmeyer 2004; Fulda et al. 2010). Although these are realistic conceptual considerations, so far there is little data on how individual cells within an entire population respond upon repeated exposure. This is largely related to the experimental 
limitations that prohibit a high dimensional analysis of the cellular stress responses at different concentrations and time points in populations of individual cells. To improve our basic understanding of cellular responses to repeated dosing scenarios, we here apply time-resolved live cell imaging of cellular stress response activation, focusing on the Nuclear factor-erythroid-2-related factor 2 (Nrf2) mediated antioxidant stress response signaling pathway.

The Nrf2 pathway plays a role in protection against chemicals with soft electrophile properties and that propagate the generation of reactive oxygen species (ROS), which may lead to oxidative stress with cell death as an ultimate outcome (Ryter et al. 2007). Such enhanced oxidative stress is typically counteracted through activation of the adaptive antioxidant cellular stress response pathway (Deshmukh et al. 2017; Ma 2013) which involves the activation of the Nrf2 pathway as the most critical component (Itoh et al. 1997; Meakin et al. 2014; Vomund et al. 2017). Nrf2 itself is a 'Cap 'n' Collar' (CNC) basic-region leucine zipper transcription factor. Under basal unstressed cellular conditions, a single Nrf2 protein is bound to two Kelchlike ECH-associated proteins (Keap1) (Keum and Choi 2014; Zipper and Mulcahy 2002). Keap1-bound Nrf2 is poly-ubiquitinated, targeting it for degradation (Kobayashi et al. 2004; Zhang et al. 2004). In response to oxidative stress and soft-electrophilic chemical exposure Keap1 is modified (Baird and Dinkova-Kostova 2013). Modification of Keap1 happens on a subset of its 27 cysteine residues (Holland and Fishbein 2010). For example, the chemicals diethyl maleate (DEM) and tert-butylhydroquinone (tBHQ) can bind to cysteine residue 151; This leads to ubiquitination of Keap1, therefore degradation of Nrf2 cannot take place (Holland and Fishbein 2010; Kobayashi et al. 2009). Current models indicate that modified Keap1 remains occupied by $\mathrm{Nrf2}$, driving accumulation of newly translated Nrf2, its translocation into the nucleus and binding and activating the antioxidant response element (ARE) in various target genes (Bryan et al. 2013; Itoh et al. 1997). Nrf2 downstream genes encode for a diverse set of adaptive programs to protect against the oxidative stress environment, exemplified by the upregulation of glutamate-cysteine ligase modifier (GCLM), heme oxygenase 1 (HMOXI), NAD(P)H quinone oxidoreductase 1 (NQO1), and sulfiredoxin1 (SRXN1). Here, we focus on the regulation of Srxn1, a bona fide Nrf2 target protein that is involved in the reduction of oxidized peroxiredoxin, a family of peroxidases which catalyzes the reduction of $\mathrm{H}_{2} \mathrm{O}_{2}$ and alkyl hydroperoxides (Chang et al. 2004; Keum et al. 2006; Soriano et al. 2008). Nrf2 pathway activation is thought to lead to an overall protection against oxidative stress, with the expectation that a similar repeated exposure would limit the antioxidant response, since sufficient protection is already available. Indeed, Nrf2 pathway activation typically leads to protection against pro-oxidant response (Itoh et al. 1997; Kensler et al. 2007; Wu et al. 2012). However, so far little is known on the dynamic modulation of Nrf2 under such repeated exposure conditions and whether limitations in the anticipated adaptation exist. Moreover, it remains unclear whether such adaptation is reflected by suppression of Nrf2 pathway activation in general or, alternatively, potential priming of the Nrf2 pathway leading to stronger pathway activation upon a repeated exposure. Understanding the outcome of diverse exposure scenarios is important for rational decision making on the safety assessment of repeated exposure.

A few reports exist on repeated exposure to known Nrf2 inducers. Mathew et al. found a stronger induction of Nrf2-dependent gene expression in primary human skin fibroblasts after repeated exposure to sulphoraphane compared to single exposure. Interestingly, an optimal concentration for repeated sulphoraphane exposure was determined that provided maximal protection against radiation injury (Mathew et al. 2014). In addition, Bergström et al. showed an ongoing accumulation of Nqo1 protein, a downstream target of the Nrf2 pathway, in astrocytes treated with $10 \mu \mathrm{M}$ sulphoraphane for $4 \mathrm{~h}$ per day over a time span of 4 days (Bergstrom et al. 2011). While these findings support different outcomes of single exposure compared to repeated exposure to Nrf2 activating agents, these studies have provided little insight into the actual behavior of Nrf2 or downstream Nrf2 target activity during repeated exposure at a single-cell level.

We have previously reported the systematic characterization and application of a panel of fluorescent protein reporters to follow individual components of the Nrf2 pathway: Keap1, Nrf2, and Srxn1 (Hiemstra et al. 2017; Wink et al. 2017, 2018). We used BAC transgenomics to tag these components with GFP and follow their behavior in individual cells over time using high-throughput confocal imaging (Wink et al. 2017). Here we applied these reporter cell lines to investigate the effect of two earlier mentioned, well-known inducers of the Nrf2 pathway, DEM and tBHQ, on the dynamics of Nrf2 and Srxn1 activation under different repeat exposure scenarios. DEM is an alkylating agent able to deplete cellular glutathione (GSH) levels by direct conjugation with GSH or via glutathione S-transferase (Casey et al. 2002; Priya et al. 2014; Yamauchi et al. 2011). tBHQ is the metabolite of butylated hydroxyanisole, a synthetic phenolic antioxidant, that acts as a redox cycler to generate ROS (Imhoff and Hansen 2010). In the present study, we used DEM and tBHQ to unravel the dynamics of Nrf2 pathway activation during repeated exposure. Our current data provide direct evidence for distinct dynamics of Nrf2 activation during a first and second treatment regimen as well as for priming of the pathway initiated during the first treatment, thus 
promoting an enhanced activation of the Nrf2 target gene $S R X N 1$ during a second treatment regimen.

\section{Methods}

\section{Chemicals}

Tert-butylhydroquinone (tBHQ, CAS: 1948-33-0), diethyl maleate (DEM, CAS: 141-05-9), L-sulphoraphane (CAS: 142825-10-3), and nitrofurantoin (CAS: 67-20-9) were obtained from Sigma-Aldrich. and Bardoxolone methyl (CDDO-Me, CAS: 218600-53-4) was obtained from Cayman Chemicals/Bio-Connect. All compounds were dissolved in 100\% dimethyl sulfoxide (DMSO, CAS: 67-68-5) from Sigma-Aldrich, to obtain aliquots with stock concentrations of $0.1 \mathrm{M}$.

\section{Cell culture}

The human hepatoma HepG2 cell line was obtained from American Type Culture Collection (ATCC ${ }^{\circledR} \mathrm{HB}-8065^{\mathrm{TM}}$, Wesel, Germany). Previously, HepG2-GFP reporter cells were developed and characterized for Nrf2 and Srxn1 (Wink et al. 2017). Briefly, cell lines were constructed with GFP reporter genes located on bacterial artificial chromosomes (BACs) that encode C-terminal GFP-tagged fusion proteins, following a selection with $500 \mu \mathrm{g} / \mathrm{mL}$ G-418. For more information, see (Poser et al. 2008). Cells were grown in Dulbecco's Modified Eagle Medium (DMEM) high glucose, supplemented with $10 \%$ $(\mathrm{v} / \mathrm{v})$ fetal bovine serum (FBS), $25 \mathrm{U} / \mathrm{mL}$ penicillin, and $25 \mu \mathrm{g}$ streptomycin. Cells were used for experiments until passage 20. Cells were seeded in 384-well plates (7000 cells/well), 2 days before exposure. Cells were exposed to concentrations in the range of $12.5-200 \mu \mathrm{M}$ of DEM or tBHQ, 2.5-50 $\mu \mathrm{M}$ sulphoraphane, 15.6-250 $\mu \mathrm{M}$ nitrofurantoin, and $25-500 \mathrm{nM}$ CDDO-Me. DMSO $(0.1 \% \mathrm{v} / \mathrm{v})$ and DMEM were used as negative controls.

\section{Cell treatment and repeated exposure scenarios}

Two different repeated exposure scenarios were used. In scenario 1, 8-h first exposure was followed by 8 -h second exposure, i.e., in total $16 \mathrm{~h}$ of live cell imaging. In scenario 2, 24-h first exposure was followed by 24-h second exposure, i.e, in total $48 \mathrm{~h}$ of live cell imaging (Fig. 1a). For both scenarios we used 9 different concentrations of DEM and tBHQ $(12.5,25,50,75,100,125,150,175,200 \mu \mathrm{M})$. All possible combinations of concentrations in the first and second exposure were tested. Furthermore, scenario 2 was also used to test the effect of repeated exposures for five different concentrations of sulphoraphane $(2.5,5,10,25,50 \mu \mathrm{M})$, nitrofurantoin $(15.6,31.3,62.5,125,250 \mu \mathrm{M})$, and CDDO$\mathrm{Me}(25,50,100,250,500 \mathrm{nM})$. In these experiments, we employed the same concentration of the compound during the second as during the first exposure.

\section{siRNA transfection}

siGENOME SMARTpool siRNAs were obtained from Dharmacon: siKEAP1, siNFE2L2, siSRXN1, siMAFF, and siMAFG. Upon arrival, siRNAs were resuspended following the manufacturer's description. siRNAs were diluted in 1 $\mathrm{x}$ siRNA buffer (Dharmacon, USA) to a final concentration of $1 \mu \mathrm{M}$. $5 \mu \mathrm{l}$ siRNA solution/well (96-well plate) was used. Interferin (Westburg/PolyPlus, NL) was used as a transfection agent. Srxn1-GFP HepG2 cells were seeded and transfected in a 96 -well plate $(23,000$ cells/well). $72 \mathrm{~h}$ after transfection, cells were exposed to the different chemicals as described above followed by high content imaging.

\section{Confocal microscopy}

Live cell confocal imaging was performed on a Nikon Eclipse Ti confocal microscope equipped with four lasers: 408, 488, 561, and $633 \mathrm{~nm}$. A 20× dry PlanApo VC NA 0.75 was used. 384 -well microclear imaging plates (microclear, Greiner) were seeded with 7000 cells/well. Prior to exposure, Hoechst ${ }_{33342} 100 \mathrm{ng} / \mathrm{mL}$ was added to the wells to stain nuclei. Subsequently, Hoechst-containing medium was washed away and medium $(25 \mu \mathrm{L})$ that contained $100 \mathrm{nM}$ propidium iodide (PI) was added to allow measurement of cell death during imaging. $25 \mu \mathrm{L} /$ well of compound containing medium was added to wells. Images were taken every hour for the indicated time periods.

\section{Temp0-Seq transcriptome analysis}

HepG2-WT cells were plated in 96-well plates (20,000 cells/ well) and exposed to $100 \mu \mathrm{M}$ DEM or tBHQ, with three independent biological replicates. After 24-h exposure, the plates where washed with $200 \mu \mathrm{l}$ PBS and lysed with $50 \mu \mathrm{l}$ BioSpyder $1 \times$ lysis buffer for $15 \mathrm{~min}$ at room temperature. After this step, plates were frozen at $-80{ }^{\circ} \mathrm{C}$. Next, the lysate plates were shipped on dry ice to BioSpyder technologies where the TempO-Seq assay was conducted (Yeakley et al. 2017). Returned gene transcription data were further analyzed using the Deseq2 package in $\mathrm{R}$ allowing to calculate the $\log 2$ fold change and the corresponding standard error $(l f C S E)$, respectively, to the base line value (medium only, no treatment). 
(A)

(C)

Nrf2 (at 8 h)

GFP/Hoechst
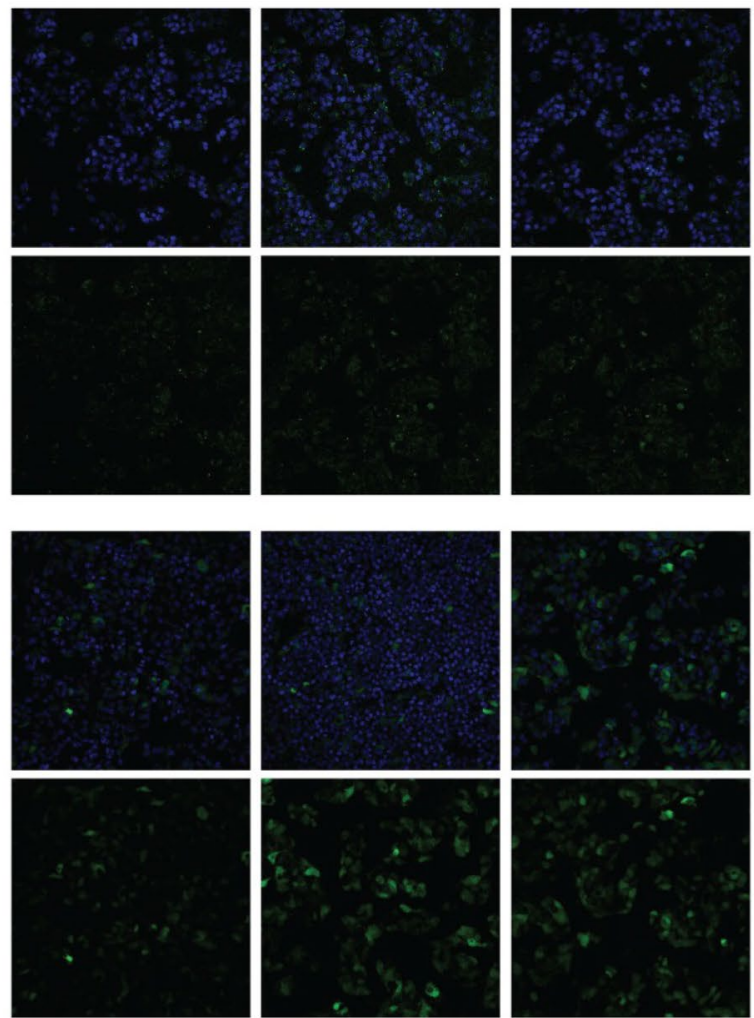

$25 \mathrm{uM}$

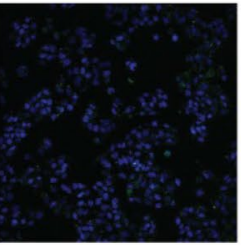

(B)
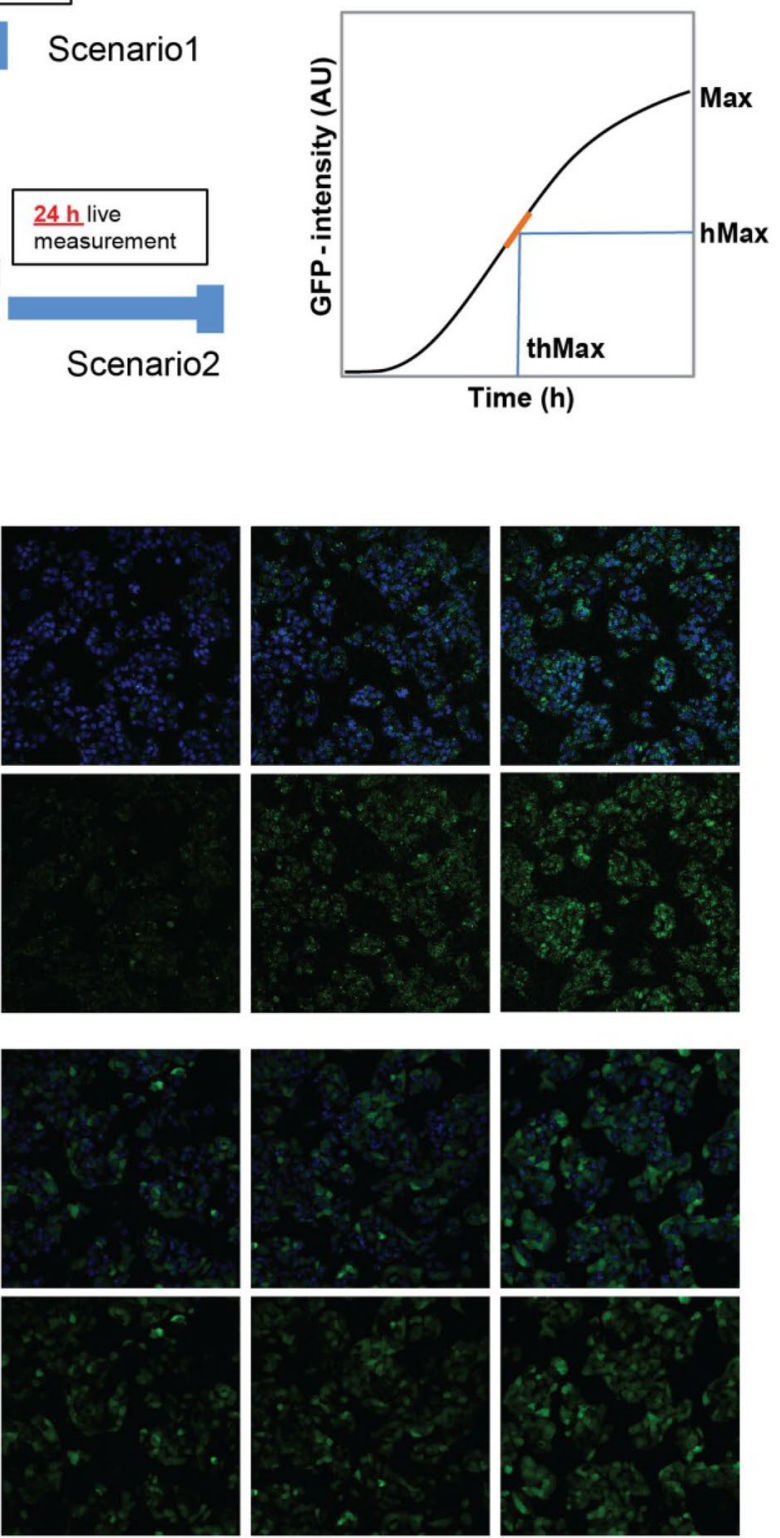

$50 \mathrm{uM}$

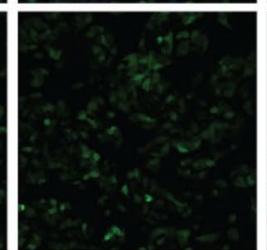

$100 \mathrm{uM}$

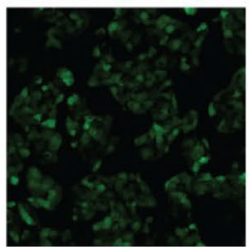

$200 \mathrm{uM}$

\section{DEM concentration}

Fig. 1 Schematic representation of the in vitro exposure scenarios and metrics. a Schematic overview of the two exposure scenarios. b Illustration of the metrics used for comparison of the dynamic behavior between the first and second exposure: Max highest value reached within experimental time frame. hMax half of the Max value, thMax time to reach hMax. The thMax is calculated by interpolating the two data points closest to half of the Max value (illustrated by

\section{Image processing and analysis}

Cell segmentation and quantification was performed with CellProfiler version 2.1.1 (Hiemstra et al. 2017; Kamentsky et al. 2011). To segment the nuclei from the orange line). c Confocal microscopy images of the Nrf2-GFP signal (at $8 \mathrm{~h}$ ) and the Srxn1-GFP signal (at $24 \mathrm{~h}$ ) after exposure to different concentrations of DEM with and without nuclear staining (Hoechst). Data are normalized per experiment and error bars depict the SEM, based on $n=3$ (for Nrf2-GFP) and $n=4$ (for Srxn1-GFP) experiments. $A U$ arbitrary units. (Color figure online)

background and each other, we used an ImageJ plugin for CellProfiler based on watershed masked clustering (WMC) as described before (Yan and Verbeek 2012). In brief, the method consists of three steps. First, the image is divided into intensity regions and starting from local maximum 
intensities, the watershed region is expanded. Second, a weighted fuzzy C-means clustering algorithm is applied to find an optimal threshold that separates background and nucleus for this region. Third, to correct nuclei that were erroneously subdivided into two different regions (i.e., that were actually a single nucleus), the algorithm merges nuclei from adjacent regions having the same orientation. We used the output of the WMC module, i.e., the segmented nuclei areas, to quantify the intensity of nuclear Nrf2-GFP and PI. To determine the intensity of Srxn1-GFP in the cytoplasm, we applied the propagation setting in CellProfiler ("identify-secondary-objects module"), employing the nuclei as seeds. This implies that CellProfiler takes the outer border of the nuclei as starting points to go outwards in a recursive manner until pixels are no longer positive for GFP or belong to a neighboring cell. In cases with high background levels that precluded correct cytoplasmic segmentation employing the propagation setting, we used the 'distance B' setting in CellProfiler ("identify-secondary-objects module"). In this setting, the nucleus is expanded by a fixed amount of pixels (using 30 pixels for our case), and in this region the background and GFP signal is distinguished.

Nrf2-GFP-intensities of single cells are calculated by taking the mean of all the pixels in a segmented nucleus. For Srxn1, the integrated GFP-intensity in the cytoplasm has been calculated with the propagation or distance B setting. We employed min-max normalization to these values, i.e., we scaled the mean GFP-intensities of individual cells per experiment between 0 and 1 , to be able to compare the biological replicates. A cell was considered GFP-positive, when its normalized GFP-intensity exceeded a threshold equal to the third quartile of the GFP-intensity distribution of cells treated with medium (negative control) during the first exposure. To determine this GFP-intensity distribution, all cells were individually included as were all time points of the first exposure. The PI-intensity within the segmented nuclei was used to decide whether a cell was considered dead or alive, based on an analysis of PI- and Srxn1-GFP-intensities. Cells with a PI-intensity of 0.2 or higher never reached a high level of Srxn1-intensity (not shown). Therefore, 0.2 was chosen as a cut-off value, and cells above this PI-intensity were considered dead. Note that the same PI-intensity threshold was applied for the Nrf2-GFP reporter cell line.

To allow comparison of the cellular response during the first exposure with that during the second exposure, we first subtracted the mean intensity of the last time point of the first exposure from the mean intensity of the second exposure. To visualize the strength of the response, we focused on the maximum value (Max), i.e., the highest mean intensity at any time point (Fig. 1b). To quantify the speed of the response, the time to reach half of the
Max value (thMax) was calculated. We used linear interpolation to estimate the time it takes to reach the halfmaximal value (hMax). Significance is determined using a one-sided welch two sample t-test.

\section{Results}

\section{Dynamics of Nrf2 and Srxn1 activation after single treatment with DEM and tBHQ}

To assess the dynamics of Nrf2 pathway activation we used two model compounds, DEM and tBHQ, two small molecules that target cysteine residues in Keap1, leading to activation of the Nrf2 pathway (Holland and Fishbein 2010; Kobayashi et al. 2009). Here, we first systematically compared the dynamics of Nrf2 activation by DEM and tBHQ upon a single dosing regimen by using confocal microscopy to monitor the stabilization and nuclear translocation of Nrf2-GFP and subsequent induction of Srxn1GFP, a direct target gene of Nrf2 (Fig. 1c). HepG2 Nrf2GFP and HepG2 Srxn1-GFP reporter-cells were exposed to different concentrations (12.5-200 $\mu \mathrm{M})$ of DEM and tBHQ followed by live cell imaging for $24 \mathrm{~h}$ (Fig. 2a, b). Both compounds caused Nrf2-GFP stabilization and translocation into the nucleus in a concentration-dependent manner (Fig. 2a). Nrf2 reaches its hMax after approximately $2 \mathrm{~h}$ of exposure to DEM or tBHQ, independent of the concentration (Fig. 2c). Overall maximal values of nuclear Nrf2-GFP were similar for DEM and tBHQ, although at high concentrations of tBHQ a sustained nuclear presence of Nrf2-GFP was observed (Fig. 2a). As anticipated, Srxn1-GFP was activated later than Nrf2-translocation to the nucleus (Fig. 2a-c). We observed slightly higher maximum values of Srxn1-GFP-intensity after treatment with tBHQ, likely due to the sustained Nrf2 activation at high tBHQ concentration. The Srxn1-response reaches its thMax consistently after approximately $8 \mathrm{~h}$ of exposure, $6 \mathrm{~h}$ later than the thMax of Nrf2 nuclear entry (Fig. 2c). We observed a linear relationship between the maximal Nrf2- Srxn1-GFP-intensities (Fig. 2d). Moreover, this relation was compound-specific, with different slopes for DEM $($ slope $=0.48)$ and tBHQ $($ slope $=0.71)$.

\section{Dynamics of Nrf2 and Srxn1 activation after repeated dosing}

There is little understanding on how prior activation of Nrf2 allows adaptation of the cell physiology and adjustment to a secondary Nrf2 activation response. We considered two different scenarios: (1) a secondary exposure at a time point when the Nrf2 response was not yet back 
(A)

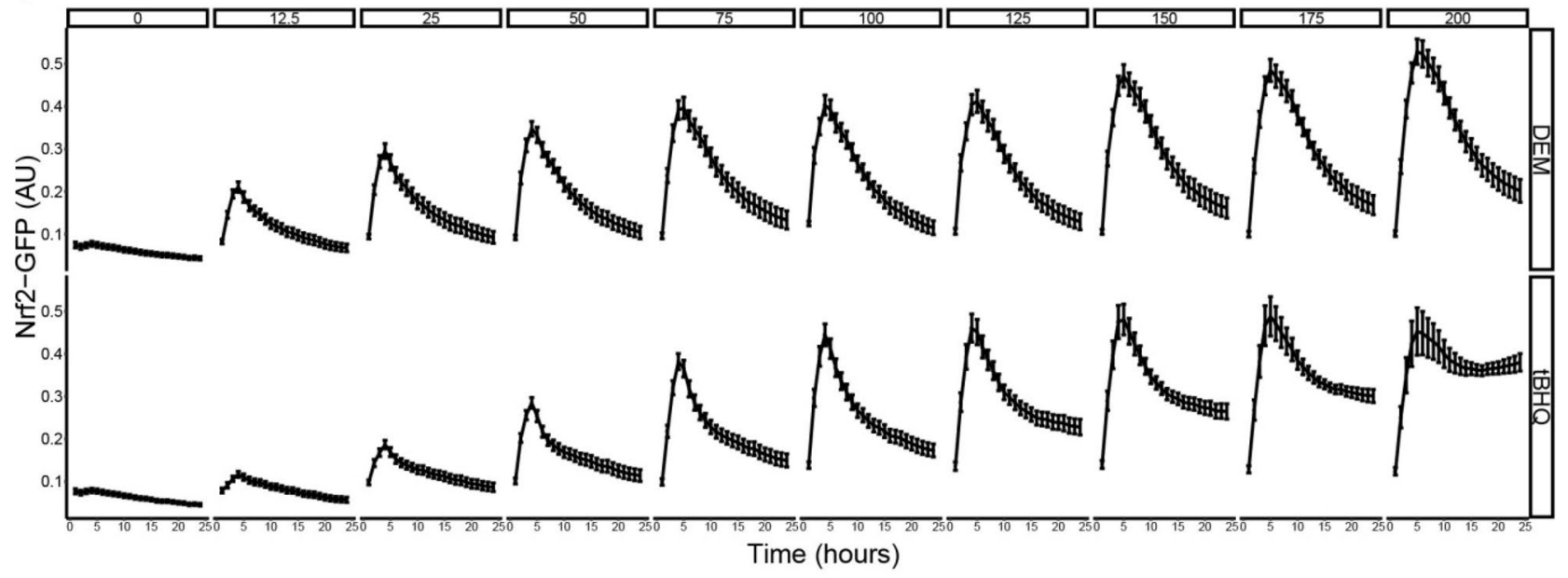

(B)

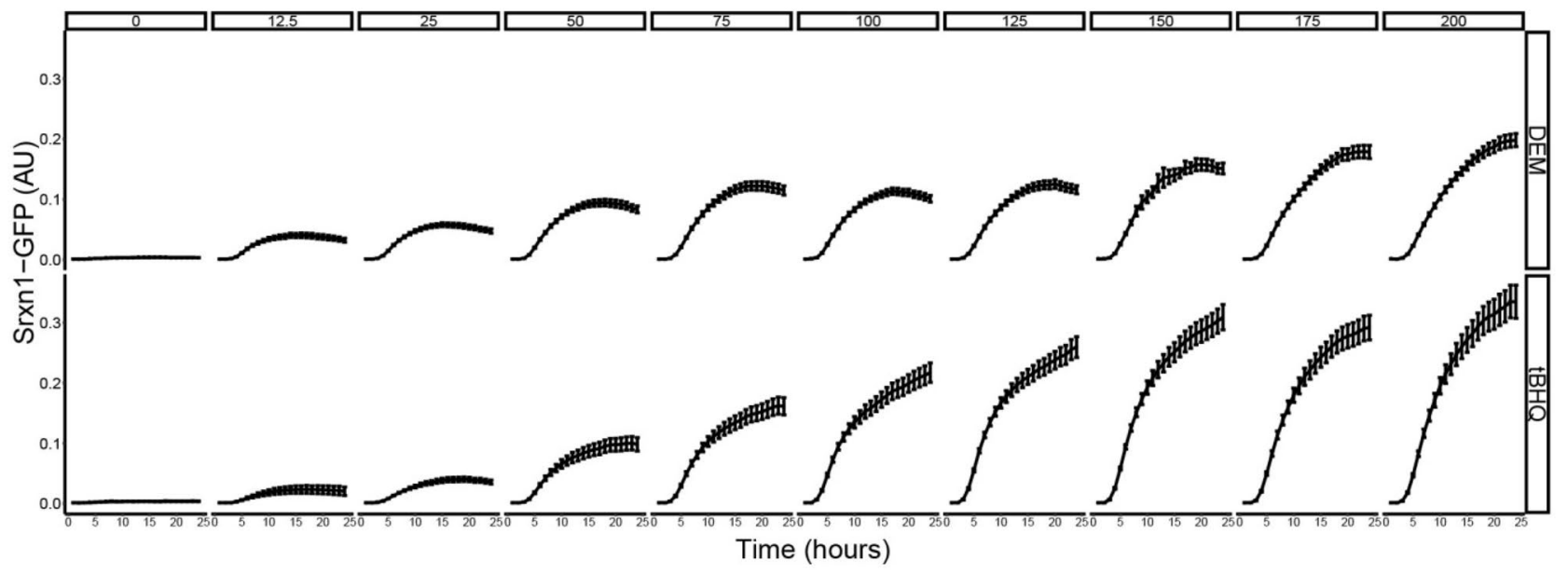

(C)

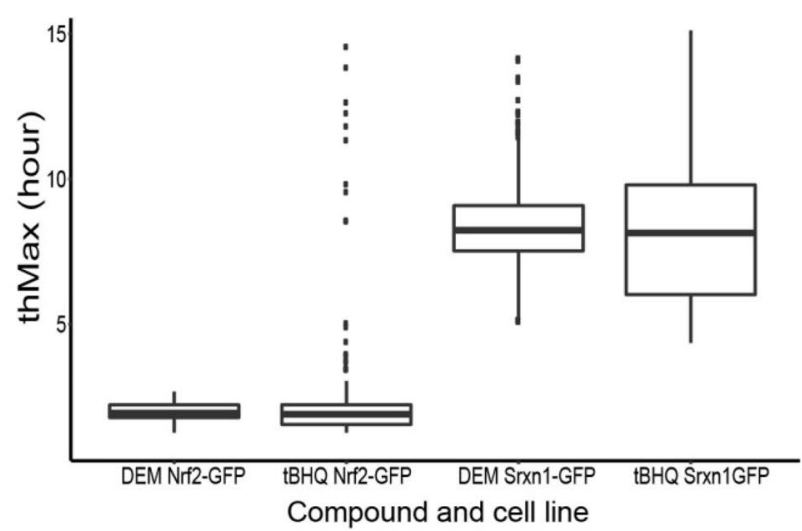

Fig. 2 Time-response curves of Nrf2 and Srxn1 activation during $24 \mathrm{~h}$ exposure to DEM or tBHQ in a dose range of 12.5-200 $\mu \mathrm{M}$. a Average Nrf2-GFP signal in the nucleus over time. b Average Srxn1GFP signal in the cytoplasm over time. $\mathbf{c}$ thMax for Nrf2 and Srxn1,
(D)

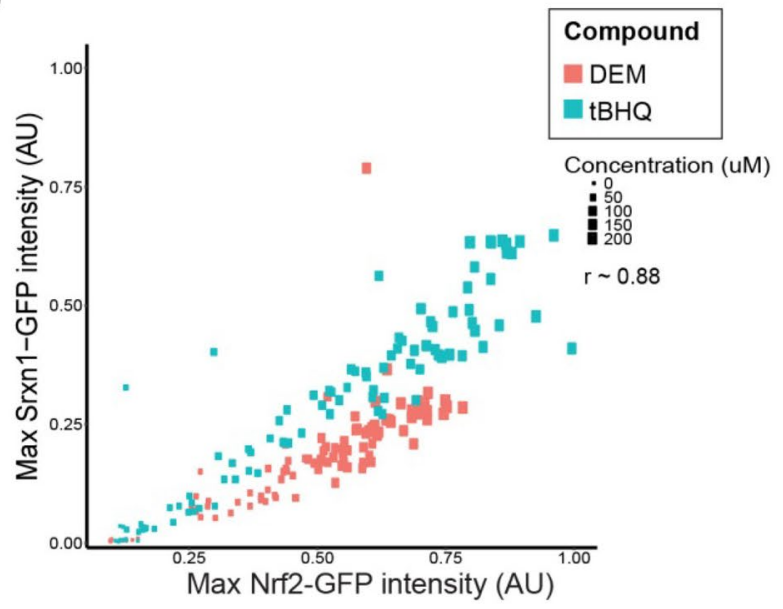

for both DEM and tBHQ. d Comparison of the maximal Nrf2-GFP and Srxn1-GFP-intensity reached after $24 \mathrm{~h}$ treatment with different concentrations of DEM or tBHQ (concentration indicated by symbol size) 
(A)

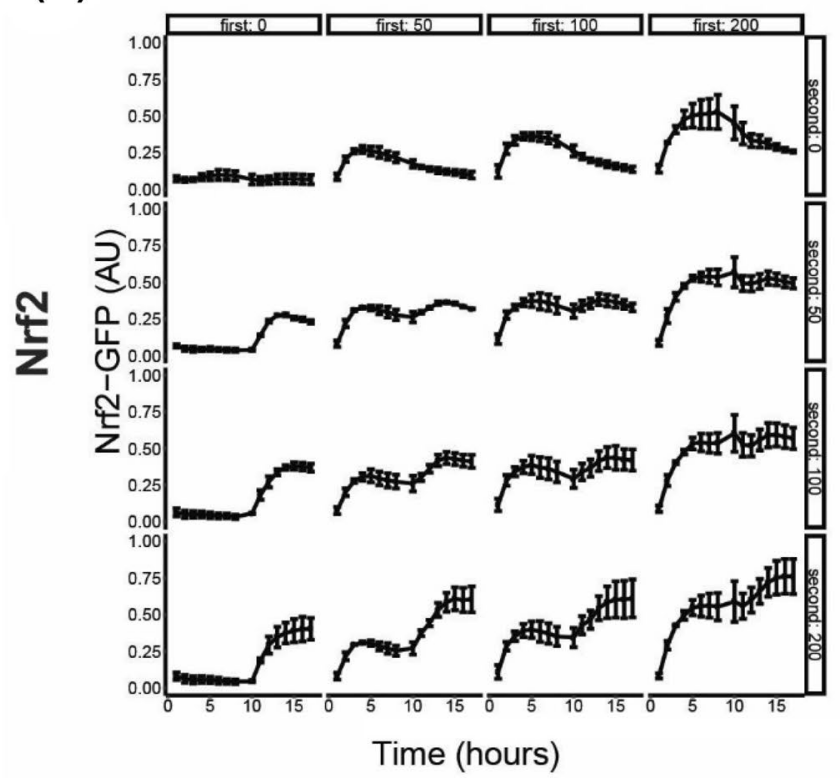

(C)

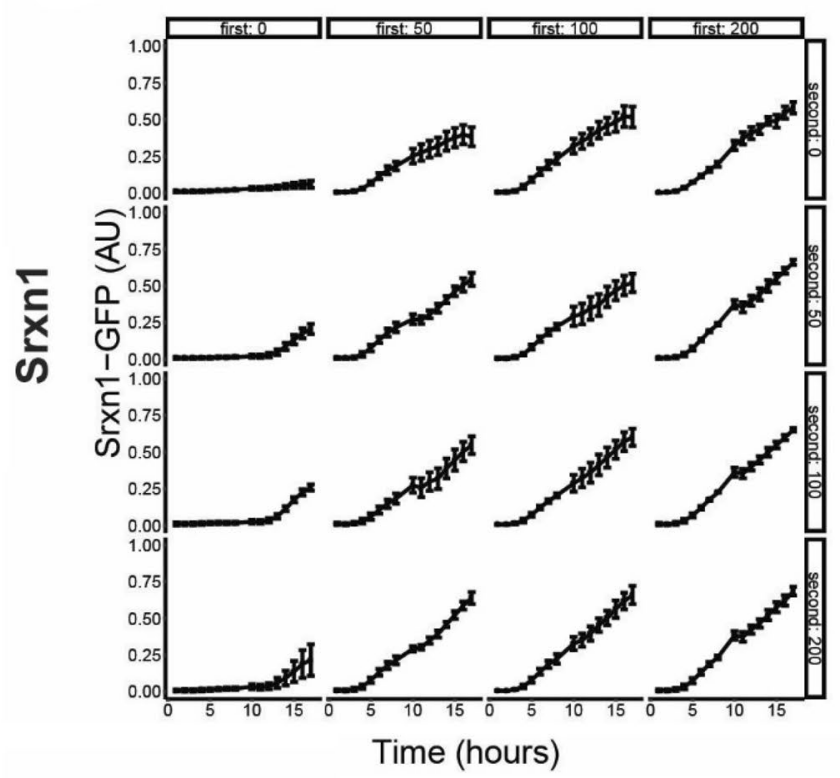

Fig. 3 Time-response curves of Nrf2 and Srxn1 activation for short repeat exposure scenario $(8 \mathrm{~h}+8 \mathrm{~h})$. Cells were exposed to DEM or tBHQ at the start and re-exposed after $8 \mathrm{~h}$ with indicated concentrations. a, b Average Nrf2-GFP-intensity in the nucleus over time after

to baseline levels and adaptation not yet fully maximal; (2) a secondary exposure at a time point when the Nrf2 response as well as the adaptation program is largely completed (see Figs. 1a, 2a, b). We first systematically evaluated the first scenario and treated cells for $8 \mathrm{~h}$ with different concentrations of DEM or tBHQ followed by a second exposure matrix at identical concentrations (Fig. 3). As
(B)

tBHQ

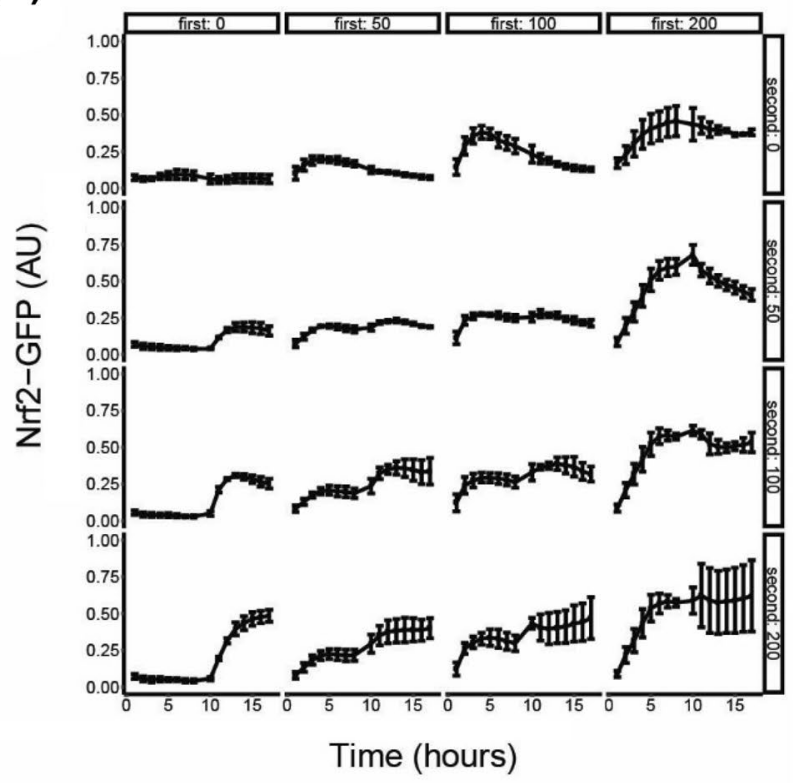

(D)

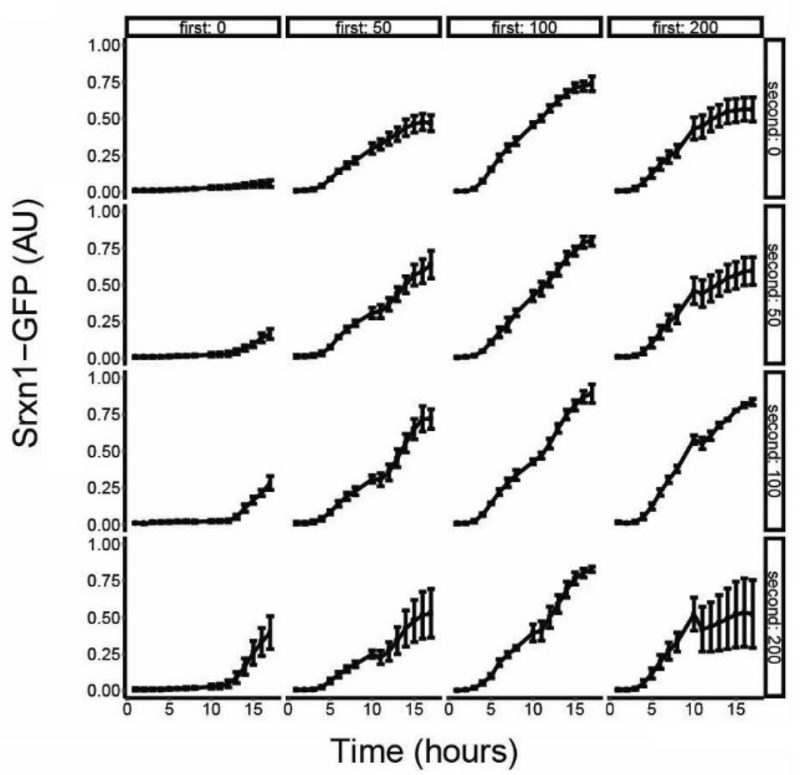

exposure to DEM (a) and tBHQ (b). c, d Average Srxn1-GFP-intensity in the cytoplasm over time after exposure to DEM (c) and tBHQ (d). Data are normalized per experiment and error bars depict the SEM; based on $n=3$ experiments

for our initial experiment with single dosing (see Fig. 2), the Nrf2-GFP response showed a peak after approximately $2 \mathrm{~h}$ and subsequently slightly declined (Fig. 3a, b). Interestingly, a second treatment after $8 \mathrm{~h}$ caused a further (re)activation of the Nrf2-GFP response, in particular when the first concentration was lower than the second concentration. However, this was not so strongly 
observed when the cells were first treated with the highest concentration of $200 \mu \mathrm{M}$, suggesting that the physiological response to activate the Nrf2 pathway was already saturated under this condition (Fig. 3a, b). The enhanced activation of Nrf2-GFP after a second exposure did not have major consequences for the activation of the downstream target Srxn1 (Fig. 3c, d). Thus, although after $8 \mathrm{~h}$ from the first treatment the Srxn1-GFP induction was already initiated, a second treatment only marginally affected

(A)
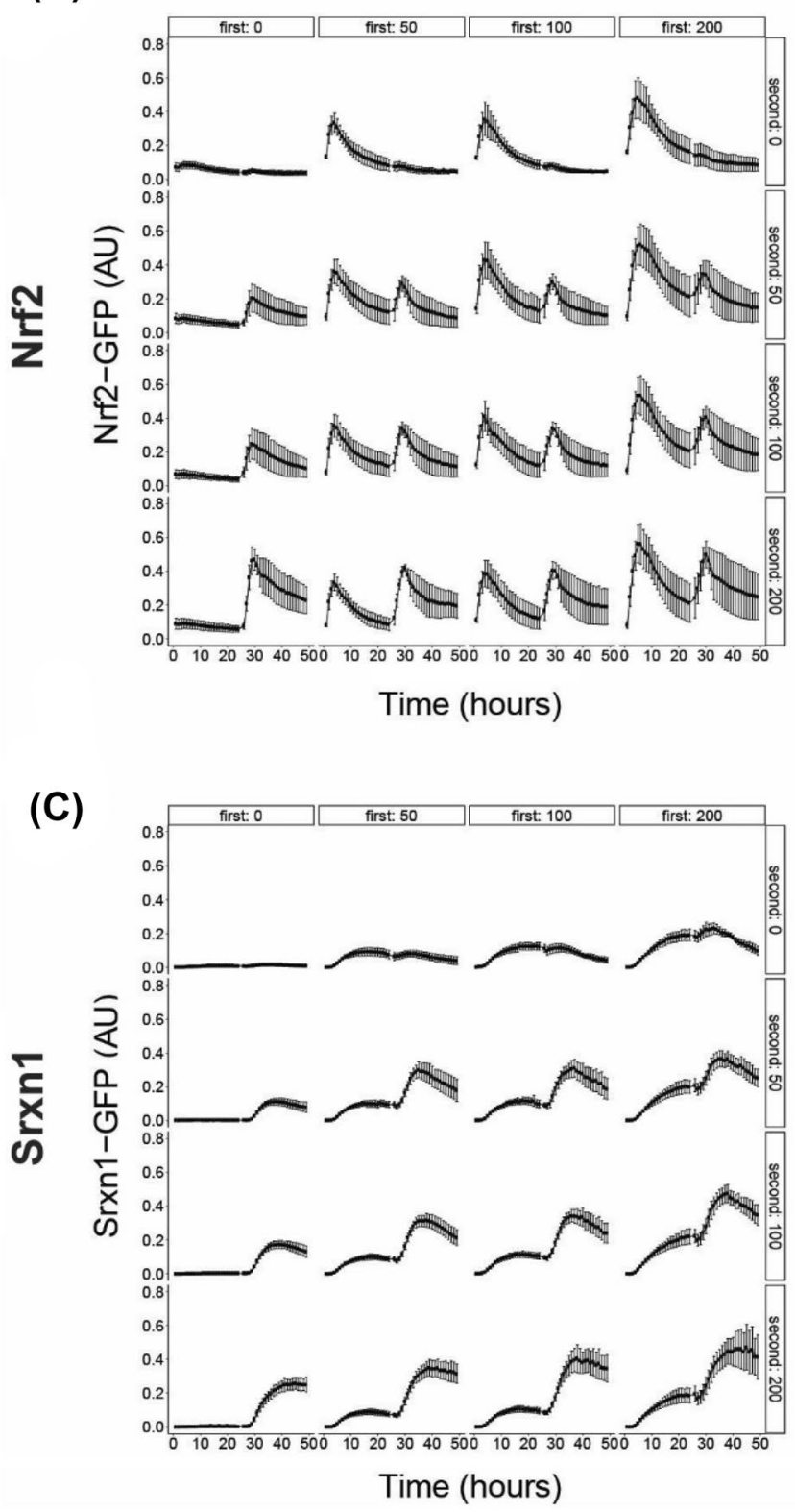

Fig. 4 Time-response curves of Nrf2 and Srxn1 activation for long repeat exposure scenario $(24 \mathrm{~h}+24 \mathrm{~h})$. Cells were exposed to DEM or tBHQ at the start and re-exposed after $24 \mathrm{~h}$. a, b Average Nrf2GFP signal in the nucleus over time after exposure to DEM (a) and the Srxn1-GFP induction, in spite of the doubling of the Nrf2-GFP response for some concentration pairs (e.g., the 50-200 $\mu \mathrm{M}$ combination for DEM). These data suggest that the 8-h repeated exposure scenario does not initiate clear adaptation, neither for DEM nor for tBHQ treatment.

Next, we determined the effect of repeated exposure at $24 \mathrm{~h}$ intervals (scenario 2). We monitored the Nrf2-GFP and Srxn1-GFP response for $48 \mathrm{~h}$, with second treatment initiation at $24 \mathrm{~h}$. The same concentration matrix for the first

(B)

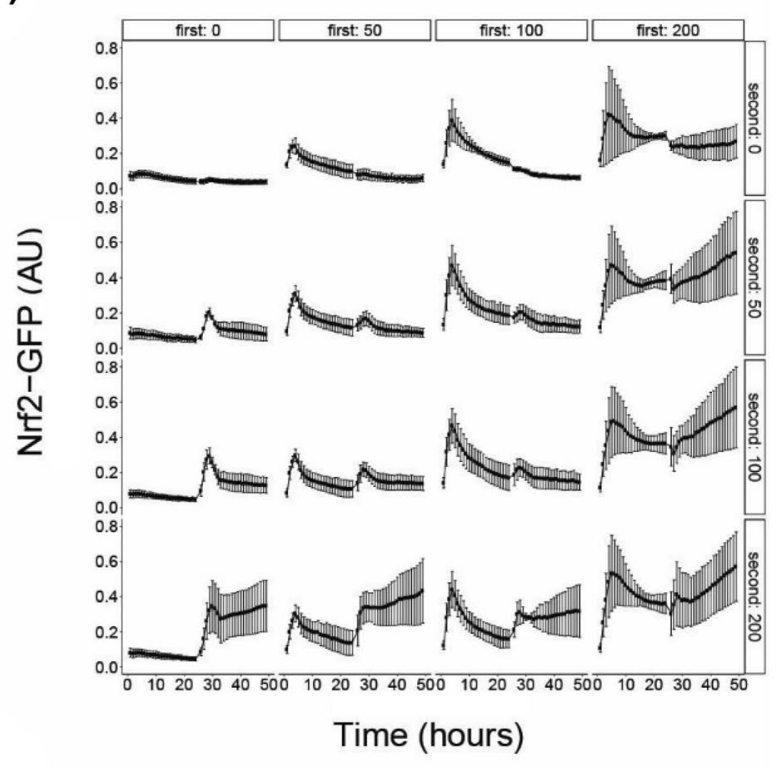

(D)

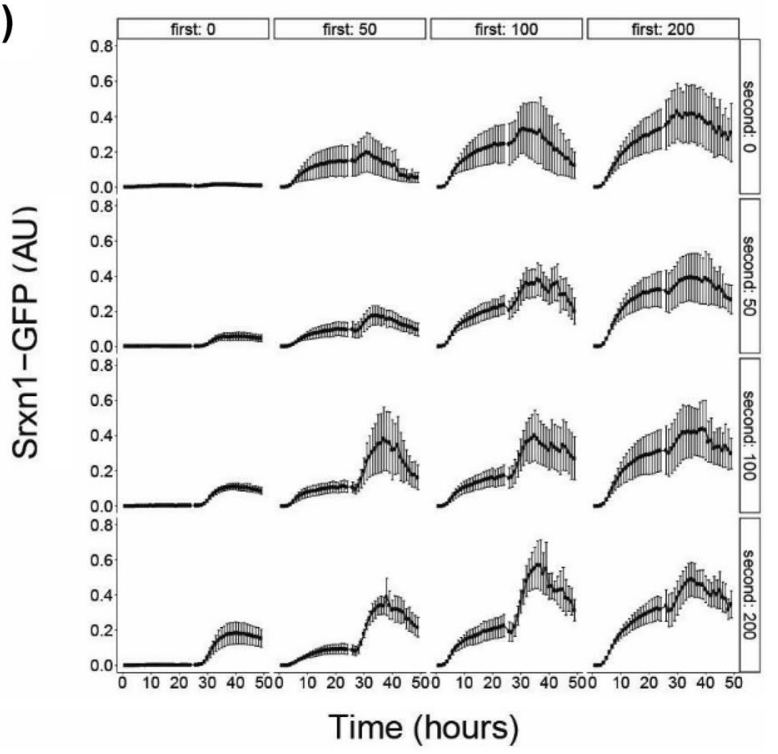

tBHQ (b). c, d Average Srxn1-GFP signal in the cytoplasm over time after exposure to DEM (c) and tBHQ (d). Data are normalized per experiment and error bars depict the SEM; based on $n=3$ (for Nrf2GFP) and $n=4$ (for Srxn1-GFP) experiments 
and second treatment was used as for scenario 1 . For both DEM and tBHQ we observed a suppression of the Nrf2GFP response when identical concentrations for first and second treatment were considered (see, e.g. $50 \mu \mathrm{M} / 50 \mu \mathrm{M}$ and $100 \mu \mathrm{M} / 100 \mu \mathrm{M}$ repeat dosing scenarios in Fig. $4 \mathrm{a}, \mathrm{b}$ ). When the concentration of the second treatment was higher than for the first treatment, a Nrf2-GFP response equal to or higher than the first response was observed. This was in particular the case for $\mathrm{tBHQ}$, where the highest concentration of tBHQ caused a sustained activation of Nrf2-GFP during the second exposure (Fig. 4b). This response of Nrf2-GFP was not reflected in the behavior of its target Srxn1-GFP. In general, despite the suppression of the Nrf2-GFP response, the Srxn1-GFP of the second treatment was stronger compared to the first treatment, both with respect to response rate as well as the amplitude of the Srxn1-GFP response (Fig. 4c, d). Only the highest concentration of tBHQ did not demonstrate such a strong secondary Srxn1-GFP response (Fig. 4d). This was likely related to the fact that a secondary treatment with $200 \mu \mathrm{M}$ tBHQ caused cell death in $~ 25 \%$ of the cells (Supplementary Fig. 1), indicating that this concentration was close to the tipping point towards onset of cell death. This coincided with the sustained accumulation of Nrf2-GFP in the nucleus. In conclusion, these data suggest that activation of the Nrf2 pathway response results in an adaptation of Nrf2 activation, in particular at late time points. In addition, such adaptation of Nrf2 activation does not imply suppression of downstream target genes of Nrf2. Intriguingly, the observed adaptation is in fact associated with an enhanced induction of Srxn1-GFP, irrespective of suppression of Nrf2-GFP activation.

\section{Population dynamics of Nrf2 and Srxn1 activation during repeated exposure}

So far, our results have demonstrated the effect of repeated treatment at the entire population level. The strength of our live cell imaging approach is that we can determine the commitment of individual cells within the entire population during both the first and second treatment with DEM and tBHQ. Therefore, next we asked whether a difference in the response during the repeated exposure was related to differences in the overall commitment of individual cells into the stress response activation. For this purpose, we determined a background GFP-threshold value based on measurements under control situations. We considered cells to be committed to the response when the GFP-values exceed this background threshold. We observed that there was a drastic commitment of more than $90 \%$ of the cells with respect to Nrf2-GFP activation within the first $2 \mathrm{~h}$ after the first treatment with the various concentrations of DEM and tBHQ. The fraction of committing cells then slowly declined to baseline levels over time if there was no second exposure.
For the second treatment we observed an equally fast increase in individual cell commitment, and prior treatment hardly affected the commitment of cells (Fig. 5), despite that the population-level amplitude of activation was lower compared to the first treatment (see Fig. 4). The commitment to Nrf2-GFP activation was typically shorter in duration for the second than for the first exposure (Fig. 5a, b). Interestingly, for high concentrations of tBHQ the overall commitment of Nrf2-GFP activation remained high.

With respect to the Nrf2-mediated activation of the Srxn1-GFP response, almost all cells committed already during the first treatment period, with a lag phase of up to $4 \mathrm{~h}$ (Fig. 5c, d). The second treatment did not further affect the commitment of individual cells into the response, besides that the overall amplitude of the Srxn1-GFP response was higher (compare Figs. 4, 5). Together, these data indicate that at the individual cell level a clear adaptation of Nrf2GFP activation occurs, where the overall commitment to Nrf2-GFP activation is sustained for a shorter period in the second treatment compared to the first treatment period.

\section{Differential activation dynamics for Nrf2 and Srxn1 activation for first and second treatment regimens}

The analysis above indicated differences in the commitment of cells with respect to Nrf2 activation. Since in our experimental setup we recorded the reporter activities for all treatment conditions with $\sim 1$-h time resolution, we next extracted the maximal Nrf2-GFP and Srxn1-GFP activation and the speed of activation onset during the first and second exposure. The maximum intensity of Nrf2-GFP for the first $24 \mathrm{~h}$ exposure was slightly higher than for the second $24 \mathrm{~h}$, supporting adaptation of the Nrf2 response (Fig. 6a). In contrast, the maximum response for Srxn1-GFP was up to three times higher after the second treatment (Fig. 6b). We also considered potential differences in the dynamics of both Nrf2-GFP and Srxn1-GFP activation. The thMax was hardly affected by concentration or compound, except for the highest repeat concentrations of tBHQ at which the Nrf2 pathway does not recover and the concentration of $\mathrm{Nrf} 2$ in the nucleus increases for the entire $48 \mathrm{~h}$ treatment duration (Fig. 6c, d). Interestingly, despite similar Nrf2-GFP dynamics between the first and second exposure, the thMax for Srxn1-GFP activation declined by almost a factor two, from $\sim 8$ to $\sim 4 \mathrm{~h}$. This is consistent with the notion that the enhanced Srxn1 upregulation is part of an adaptation program to control prolonged exposure to soft electrophiles such as DEM and tBHQ. Because of the opposite direction of the response, i.e. the reduced response of Nrf2-GFP and the increased response of Srxn1-GFP during the second treatment condition, the linear relationship between Nrf2-GFP and Srxn1-GFP activation that we observed for 


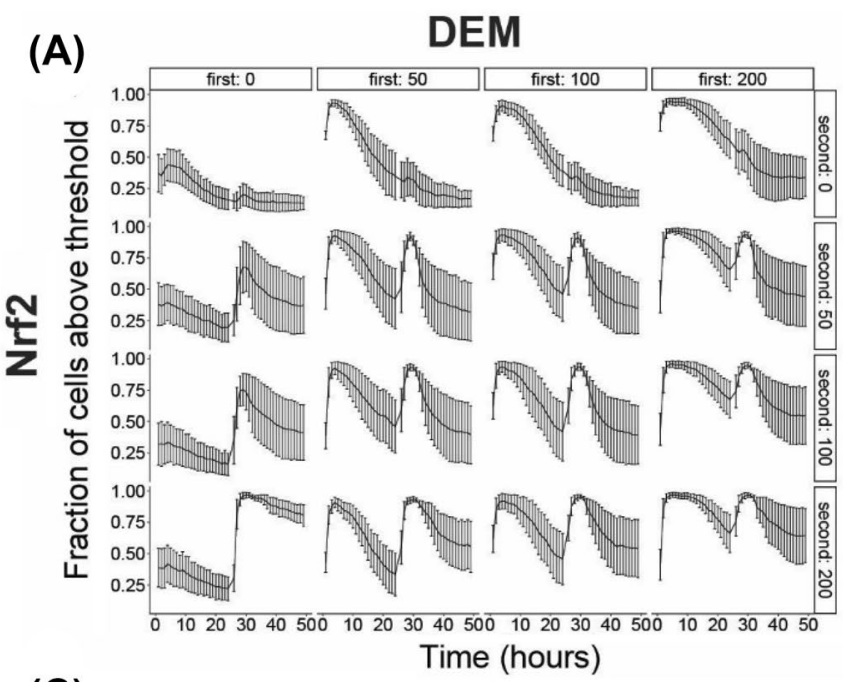

(C)

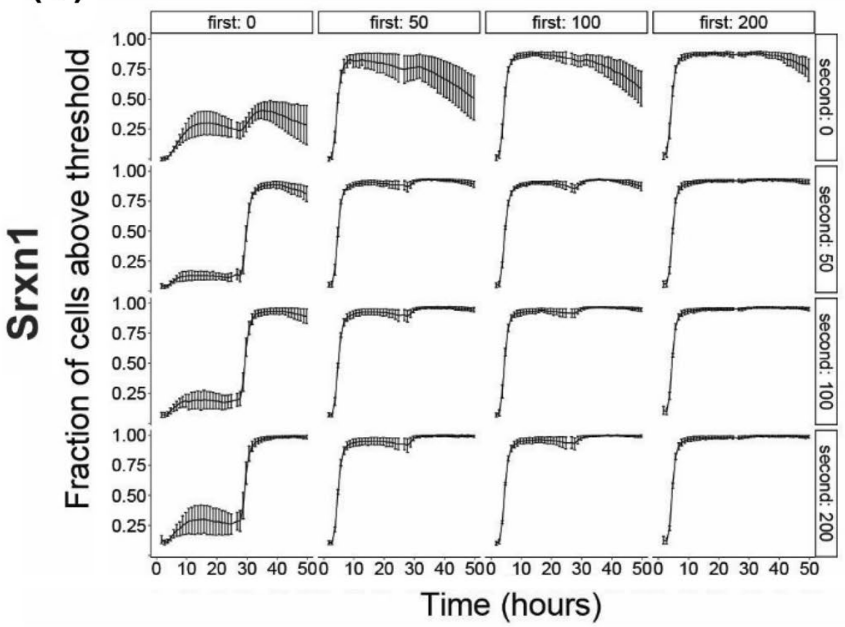

Fig. 5 Commitment of individual cells into the Nrf2 and Srxn1 response during long repeat exposure scenario. Cells were exposed to DEM or tBHQ at the start and re-exposed after $24 \mathrm{~h}$. a, b Fraction of cells whose nuclear Nrf2-GFP levels exceed background levels over time, after exposure to DEM (a) and tBHQ (b). c, d Fraction of cells

the first exposure was less clearly present for the second treatment period (Fig. 6e). Moreover, the slope difference between DEM and tBHQ was no longer apparent. These data together indicate that during repeated treatment with soft electrophilic chemicals, different mechanisms take part in the cellular stress response activation of the Nrf2 pathway for the first and for subsequent exposures.

\section{Secondary enhanced activation of Srxn1 expression is dependent on the Keap1/Nrf2 pathway}

We next investigated the underlying mechanism of the Srxn1 induction during repeated exposure. Because $S R X N 1$ is a well-described Nrf2 target gene (Soriano et al. 2008), we investigated whether both the first and second induction of

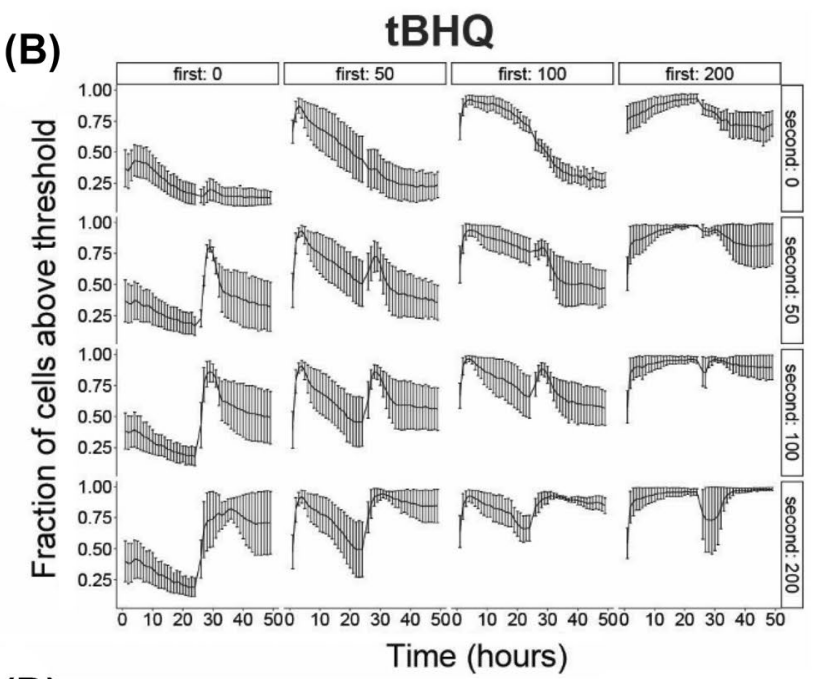

(D)

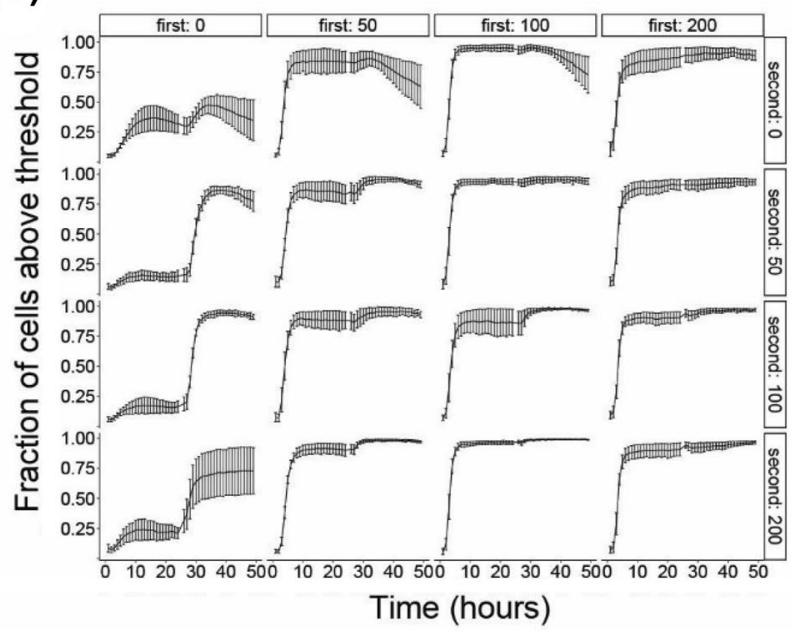

whose cytoplasmic Srxn1-GFP levels exceed background levels, after exposure to DEM (c) and tBHQ (d). Data are normalized per experiment and error bars depict the SEM; based on $n=3$ (for Nrf2) and $n=4$ (for Srxn1) experiments

Srxn1-GFP expression depended on the Keap1/Nrf2 pathway using siRNA knockdown experiments. siSRXN1 inhibited the induction of SRXN1 during both the first and the second exposure to DEM and tBHQ (Fig. 7a, b), indicating that our knockdown condition was effective. Since Keap1 targets Nrf2 for degradation, we anticipated that knockdown of KEAP1 would enhance Nrf2 activity and promote Srxn1GFP expression. Indeed, siKEAPl enhanced both the first and second induction of Srxn1-GFP by DEM and tBHQ (Fig. 7a, b). Finally, knockdown of Nrf2 itself through $s i N$ FE2L2 led to inhibition of Srxn1 induction during first and second exposure (Fig. 7a, b), demonstrating that the strong induction of Srxn 1 during the second response depends primarily on Nrf2. 
(A)

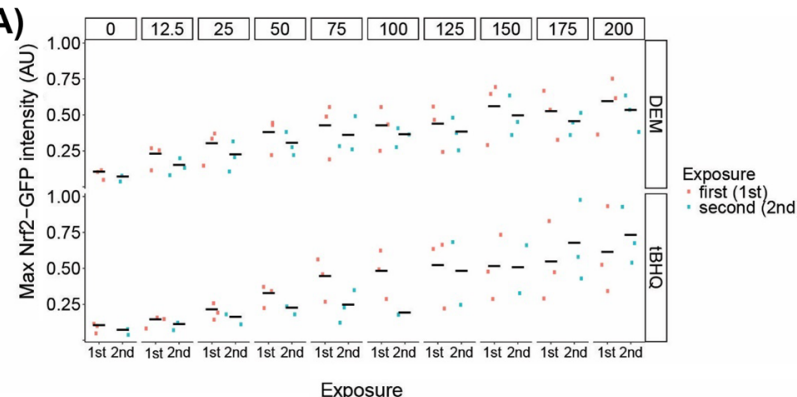

(C)

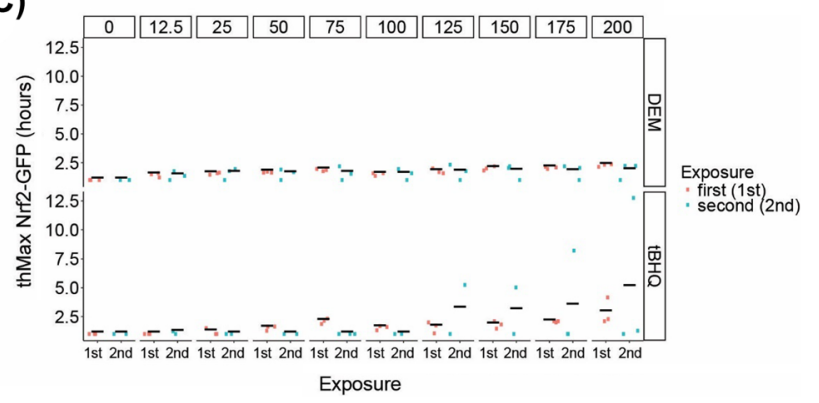

(B)

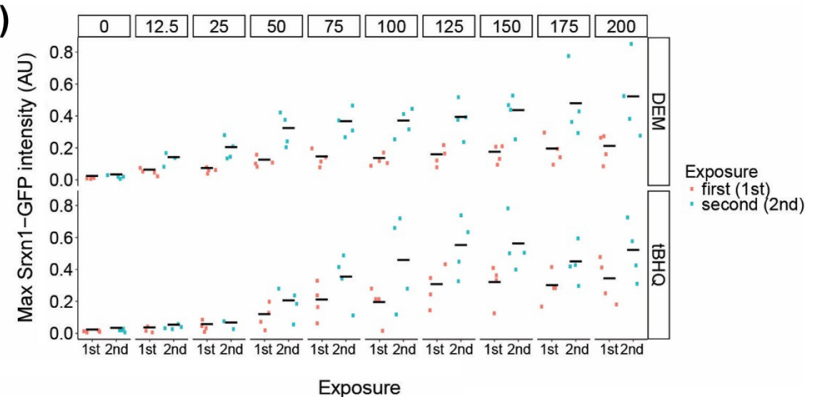

(D)

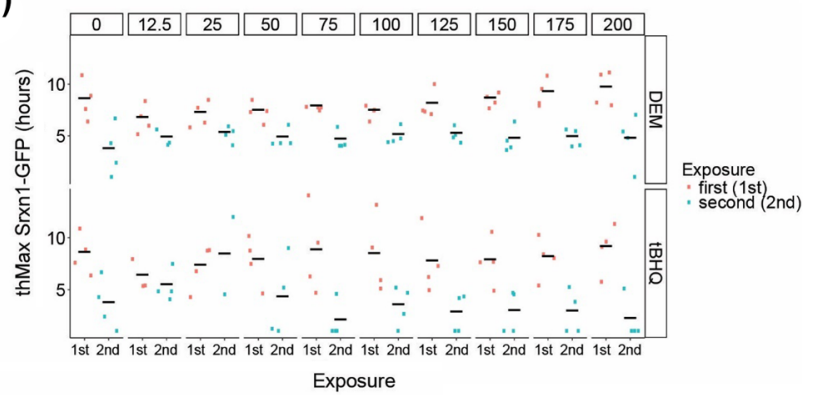

(E)

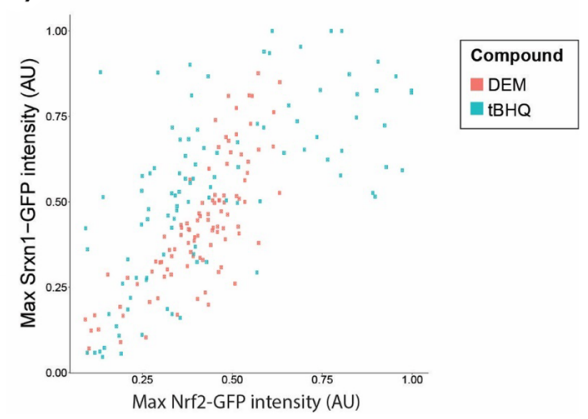

Fig.6 Quantitative analysis of Nrf2 and Srxn1 activation dynamics during first and second treatment periods. a, b The maximal values reached within $24 \mathrm{~h}$ after the first or second exposure to DEM or tBHQ for Nrf2-GFP (a) and Srxn1-GFP (b). c, d thMax after the first

MAF-transcription factors are co-activators of Nrf2 as well as downstream targets of Nrf2, offering a potential explanation for the increased Srxn1 induction during repeated exposure. However, we did not observe an induction of either $M A F F$ or $M A F G$ gene expression at $24 \mathrm{~h}$ after the first treatment with DEM or tBHQ (Fig. 7c). Moreover, knockdown of neither $M A F F$ nor $M A F G$ inhibited the induction of Srxn1-GFP during either the first or second exposure (Fig. 7d), suggesting that MAFs do not provide an explanation for the enhanced Srxn1 induction in the second exposure. Altogether, these data indicate a primary role for Nrf2 in the regulation of secondary Srxn1 induction. or second exposure to DEM or tBHQ for Nrf2-GFP (c) and Srxn1 (d). e Comparison of the maximal Nrf2-GFP and Srxn1-GFP values reached within $24 \mathrm{~h}$ after second exposure to DEM or tBHQ

\section{Enhancement of secondary Srxn 1 induction by other compounds including drugs that activate the Nrf2 pathway}

Finally, we evaluated whether the enhanced secondary response was also observed for compounds where Nrf2 activation is related to the direct pharmacology or off-target effects. Specifically, we included bardoxolone methyl (CDDO-Me) (Cleasby et al. 2014; Wang et al. 2017), sulphoraphane (Alumkal et al. 2015; Lynch et al. 2017), and nitrofurantoin (Herpers et al. 2016; Tsuchiya et al. 2018), each of which activates the Nrf2 pathway at a different concentration range. Evaluation of Srxn1-GFP expression through imaging at 24 and $48 \mathrm{~h}$ post exposure with CDDO$\mathrm{Me}(250 \mathrm{nM})$, sulphoraphane $(10 \mu \mathrm{M})$ and nitrofurantoin $(250 \mu \mathrm{M})$ demonstrated that Srxn1 was induced at $24 \mathrm{~h}$ and that this induction was further enhanced by a second 
(A)

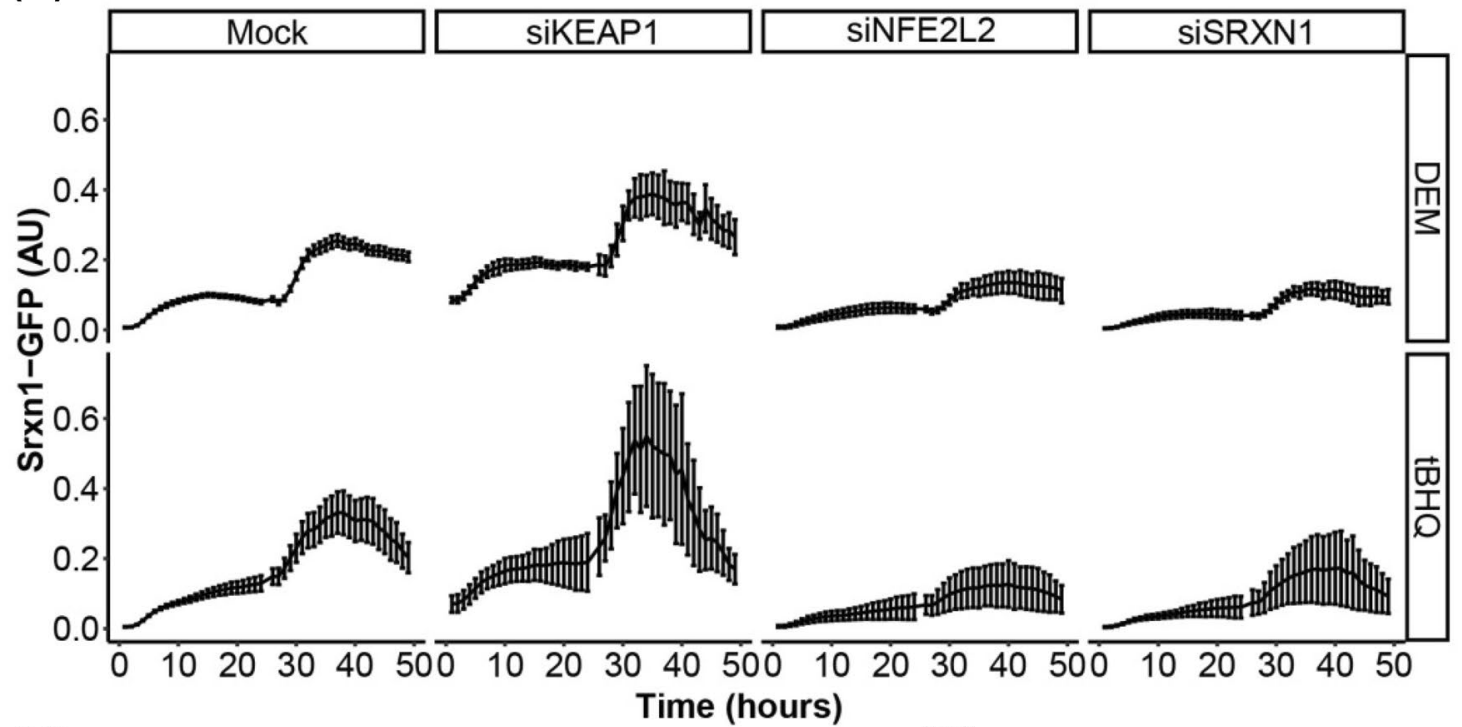

(B)

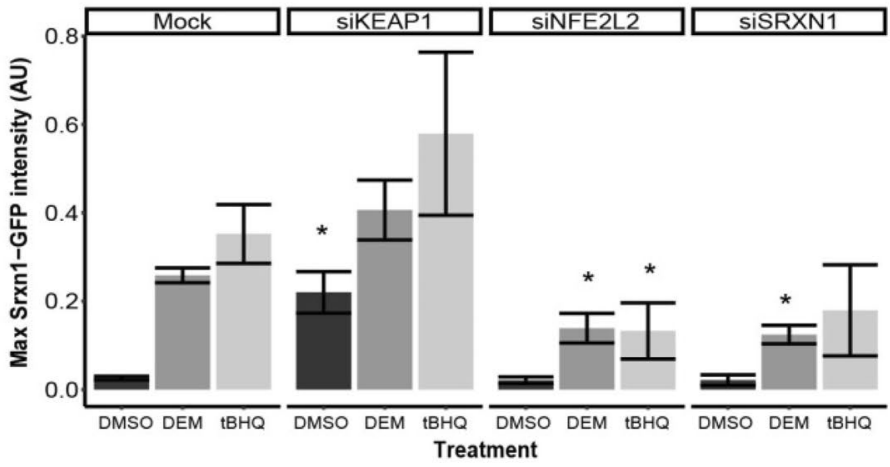

(C)

(D)

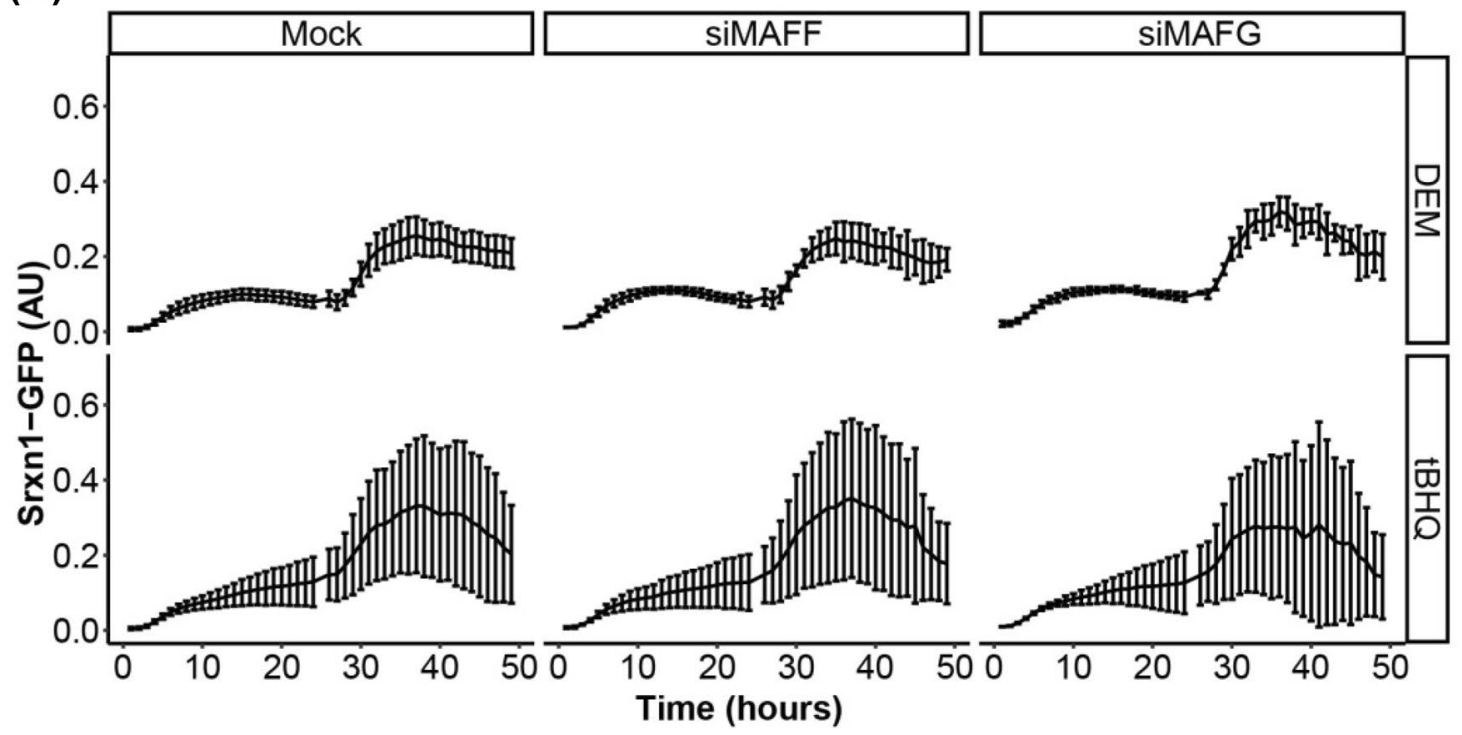


४Fig.7 siRNA mediated knockdown of different Nrf2 pathway proteins. a Time-response curves of Srxn1-GFP induction after treatment with DEM $(100 \mu \mathrm{M})$ or tBHQ $(100 \mu \mathrm{M})$ in mock condition or with siKEAP1, siNFE2L2 or siSRXN1. b Maximal Srxn1GFP-intensity at $24 \mathrm{~h}$ after exposure to DMSO (0.1\%), DEM (100 $\mu \mathrm{M})$ or tBHQ $(100 \mu \mathrm{M})$ and in mock condition or transfection with mock condition, siKEAP1, siNFE2L2 or siSRXN1. Significance is shown with $a *$ based on $a<0.05 p$ value comparing the same treatment in the mock condition, hypothesizing that the siKEAP1 knockdown gives a higher Srxn1-GFP-intensity, while the other siknockdowns have a lower Srxn1-GFP-intensity compared to mock. c Gene expression changes of MAFF, MAFG, and SRXN1 vs baseline (i.e., medium sample just before treatment) $24 \mathrm{~h}$ after exposure to DMSO $(0.1 \%)$, DEM $(100 \mu \mathrm{M})$ or tBHQ $(100 \mu \mathrm{M})$. d Time-response curves of Srxn1-GFP induction after treatment with DEM $(100 \mu \mathrm{M})$ or tBHQ $(100 \mu \mathrm{M})$ in mock condition or with siMAFF or siMAFG. Results are in all cases based on $n=3$ experiments, with error bars depicting the SEM $(\mathbf{a}, \mathbf{b}, \mathbf{d})$ or the lfcSE $(\mathbf{c})$

exposure from 24 to $48 \mathrm{~h}$, similar as for exposure to DEM (Fig. 8a, b). In contrast, a continuous single treatment with these drugs for $48 \mathrm{~h}$ did not lead to a similar high Srxn1GFP level, except for CDDO-Me, which is likely due to the prolonged response this compound causes (Wink et al. 2017). These data indicate that the enhancement of Srxn1 expression during a secondary $\mathrm{Nrf} 2$ response is in general relevant for drugs that can activate the Nrf2 pathway.

\section{Discussion}

Our general understanding of the dynamics of cellular stress response pathway activation in repeated treatment scenarios is limited. Here, we systematically mapped the Nrf2 pathway adaptive response landscape of repeated exposure to two different soft electrophiles, DEM and tBHQ. We took advantage of two biologically relevant fluorescent HepG2 reporter cell lines that allowed us to monitor the activation of Nrf2 and its downstream target Srxn1 in individual cells over time using live cell confocal imaging. Our data indicate that cells adapted to oxidative stress: During a second treatment they have limited Nrf2 activation and a relatively short-lasting commitment, yet cells are primed to exhibit enhanced activation of Nrf2 downstream target Srxn1.

Our high-throughput analysis of Nrf2-GFP and Srxn1GFP activation for diverse concentrations demonstrated that there is a linear and compound-dependent relationship between the maximal amount of Nrf2 in the nucleus after single exposure and the subsequent maximal amount of Srxn1 in the cytoplasm. Single exposure to DEM and tBHQ resulted in a concentration-dependent activation of both Nrf2 and Srxn1, with slightly different dynamics for both compounds. The different dynamics observed and the different correlation between Nrf2 and Srxn1 might be due to the different manners in which both compounds activate the Nrf2 pathway, involving both Keap1-dependent and -independent activation mechanisms (Bryan et al. 2013; Lee et al. 2001). Moreover, the half-life of DEM and tBHQ in the culture conditions may differ, offering a potential explanation for differential activation dynamics of Nrf2, although this does not explain why there is a different Nrf2 to Srxn1 ratio. The latter was in particular apparent for very high tBHQ concentrations.

For the first exposure, Nrf2-GFP reached hMax activation levels within $2 \mathrm{~h}$, which was associated with an overall commitment to Nrf2 activation of $>90 \%$ of the cells. Interestingly, when a second exposure was initiated after $8 \mathrm{~h}$ both DEM and tBHQ could further promote Nrf2-GFP accumulation, despite the fact that the response had not yet returned to baseline. Apparently, the machinery to produce newly synthesized Nrf2-GFP was not yet at its maximum capacity, and/or there was still remaining Keap1 to be targeted by the electrophiles, further suppressing Nrf2 ubiquitination. Regardless, the increased amount of Nrf2-GFP did not equally enhance Srxn1 activation, as the amount of Srxn1GFP hardly changed. Thus, adaptation to electrophiles takes longer than $8 \mathrm{~h}$, which is relevant for the design of repeated dose scenarios in the context of pharmacological modulation of the Nrf2 pathway, yet also for toxicant exposure scenarios in daily life that may cause Nrf2 pathway activation.

Two main observations indicate that adaptation at the level of Nrf2-GFP activation occurs during long-term (24 h) repeated treatment scenarios. First, the Nrf2-GFP nuclear accumulation was lower for the second treatment than for the first treatment. This effect was strongest when the same concentration was applied during the first and second treatment, but was also visible when the concentration of the second treatment was higher. Second, the overall time period that individual cells remained committed to Nrf2GFP nuclear accumulation was shorter for the second treatment. This effect was observed both for DEM and tBHQ, although for $\mathrm{tBHQ}$ the overall commitment of the population after the second treatment did not reach the levels of the first exposure. The stabilization and nuclear accumulation of Nrf2 is known to reflect the activation of the oxidative stress sensing machinery (Kobayashi et al. 2006). Thus, if pro-oxidants affect the Cys residues of Keap1 more strongly, the degradation of Nrf2 is also more difficult. Because we observed less Nrf2-GFP in the nucleus and a shorter commitment period for cells in the second compared to the first exposure, this suggests a limited targeting of the sensing machinery. Hence, during the second exposure both DEM and $\mathrm{tBHQ}$ may be more rapidly detoxified by the action of downstream Nrf2 targets, including Srxn1.

The adaptation to both DEM and tBHQ resulted in an unanticipated further upregulation of Srxn1-GFP. Thus, despite the relatively low response of Nrf2 after the second treatment, Srxn1-GFP showed a threefold increased induction. Given the critical role of Srxn1 in the antioxidant 
(A)

I.

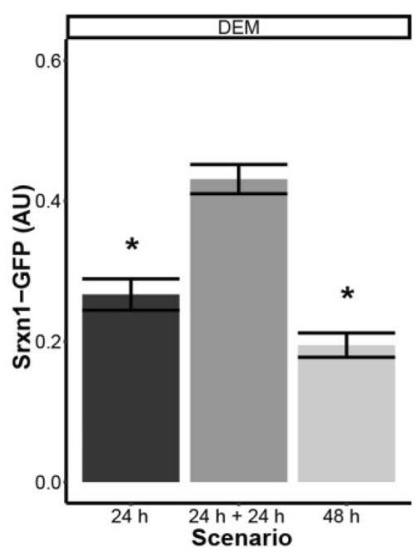

II.

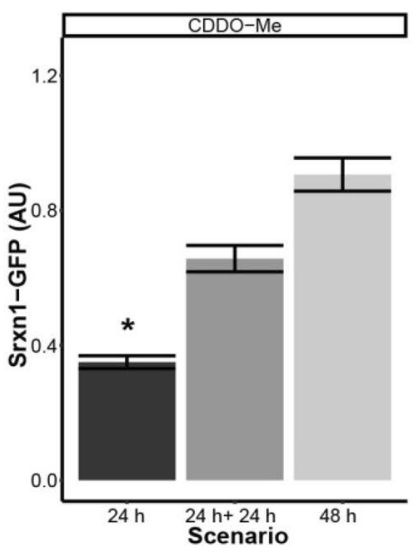

(B)

I.

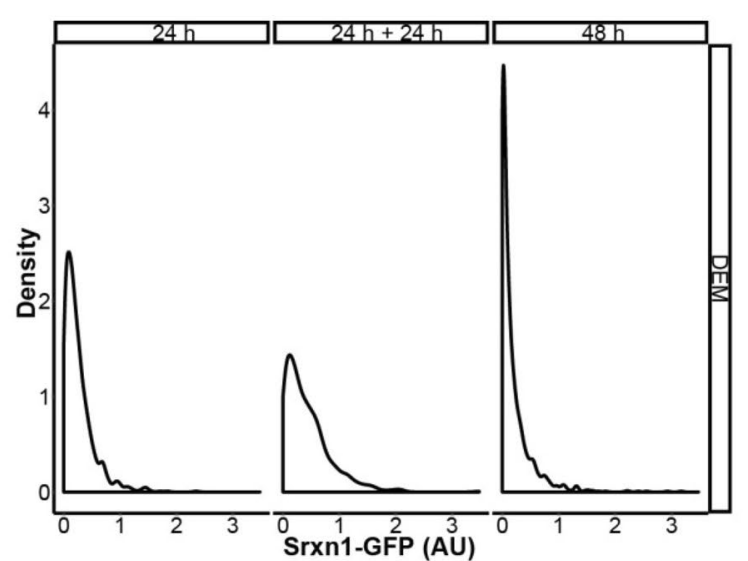

III.

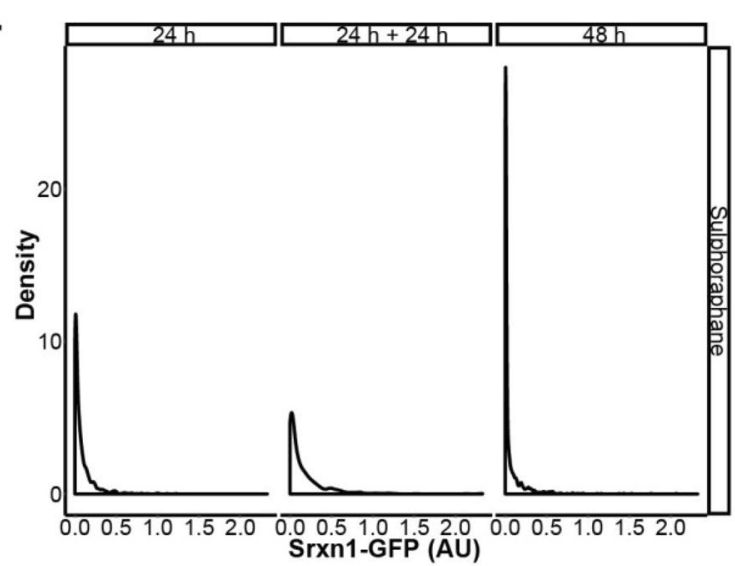

Fig. 8 Srxn1 induction following repeated exposure to various drugs. Srxn1-GFP reporter cells were exposed for either $24 \mathrm{~h}$, or exposed for two time periods of $24 \mathrm{~h}$, or for a single time period of $48 \mathrm{~h}$ to CDDO-Me $(0.25 \mu \mathrm{M})$, DEM $(50 \mu \mathrm{M})$, Nitrofurantoin $(250 \mu \mathrm{M})$, Sulphoraphane $(10 \mu \mathrm{M})$ or DMSO $(0.1 \%)$. a Srxn1-GFP expression after chemical exposure. Significance is shown with $a *$ based on $a<0.05$
III.

IV.
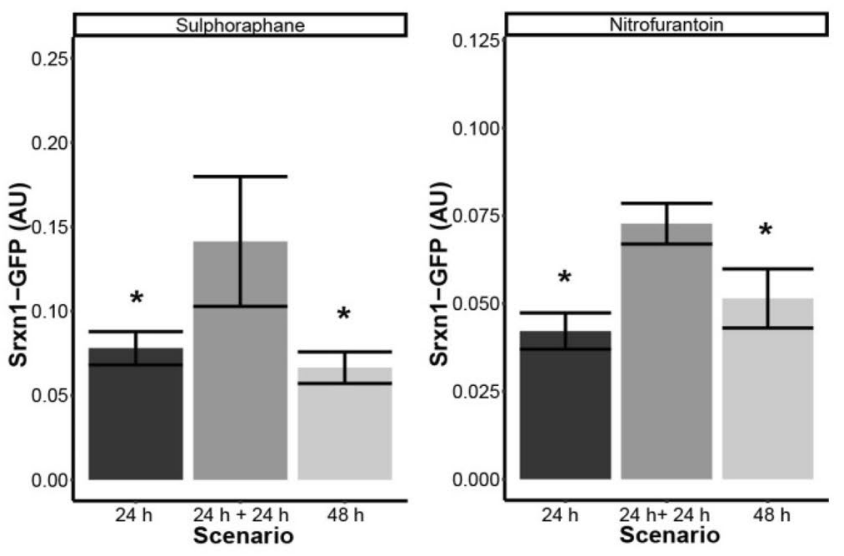

II.

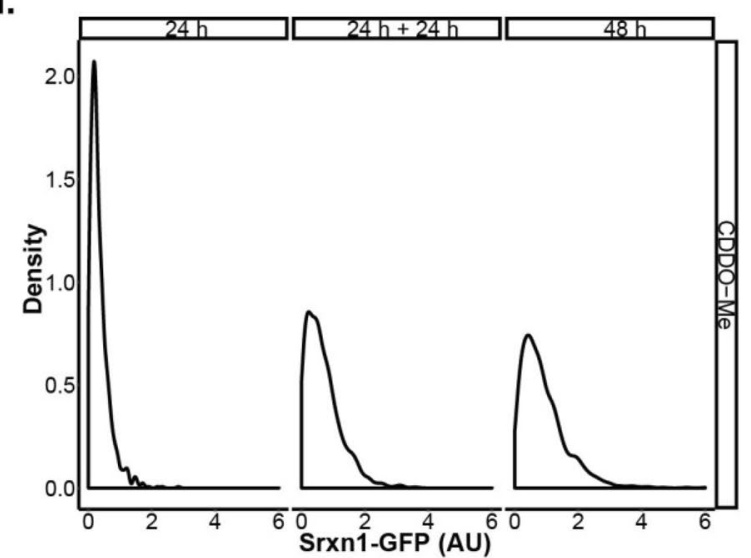

IV.

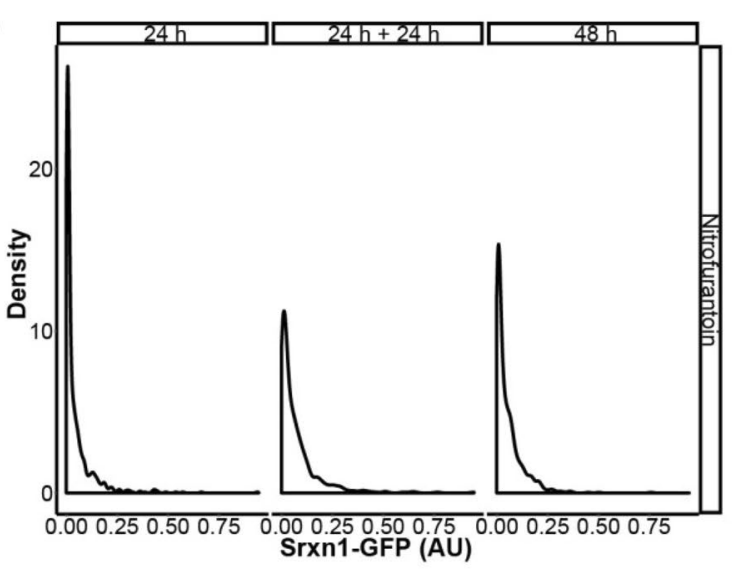

$p$ value comparing $24 \mathrm{~h}$ exposure or $48 \mathrm{~h}$ exposure to the repeated $24 \mathrm{~h}$ exposure, hypothesizing that the repeated exposure has always the highest Srxn1-GFP-intensity. b Population commitment to Srxn1 induction presented as density plot, platted as fraction of cells ( $y$ axis) expressing the Srxn1-GFP levels ( $x$ asis). Shown are average data of $n=3$ experiments 
response (Baek et al. 2012; Soriano et al. 2008; Zhou et al. 2015), we presume that this phenomenon is a critical component of an adaptation program that primes cells to subsequent exposures and improves protection against oxidative stress. The Keap1/Nrf2 interaction was the main component responsible for the enhanced secondary response, since knockdown of NFE2L2 strongly inhibited this response and knockdown of KEAP1 promoted it. Still, this enhanced response of Srxn1-GFP is likely not driven by Nrf2 alone, since the Nrf2-GFP nuclear activity was lower during the second exposure than during the first exposure. In our hands, knock-down of MAF-family transcriptional regulators $M A F F$ and $M A F G$ that can modulate Nrf2 transcriptional activity did not affect the enhanced Srxn1 induction. We, therefore, anticipate that other factors are involved in the secondary response that also themselves might be modulated as part of the primary response.

In the literature, some evidence has been presented for the accumulation of downstream targets of Nrf2 during repeated exposure. For example, Bergström et al. (2011) showed a daily accumulation of NQO1 mRNA and protein in astrocytes treated with $10 \mu \mathrm{M}$ sulphoraphane for $4 \mathrm{~h}$ per day over a time span of 4 days. Interestingly, they did not find the same for HMOX1, which only exhibited an increase after the first exposure, but no further accumulation after repeated exposure. Similar conclusions with respect to NQO1 and HMOX1 were obtained by Mathew et al. (2014), who treated human fibroblasts with different concentrations of sulphoraphane for $4 \mathrm{~h}$ per day over a time span of 3 days. To what extent these effects are related to activity of $\mathrm{Nrf} 2 \mathrm{and} /$ or other factors remains unclear. Factors that govern Nrf2 activity itself may also be relevant for the observed priming effects at the level of Srxn1-GFP induction. This could be related to post-translational modification of Nrf2 through for example phosphorylation or acetylation (Huang et al. 2000; Sun et al. 2009) or through the induction of transcriptional co-regulators that act in concert with Nrf2 to target specific genes, including for example p21 (Chen et al. 2009; Katsuoka et al. 2005). Alternatively, given the role of Keap1 in modulating Nrf2 changes in the overall Keap1/Nrf2 interactome, modifications in this interactome may also effect Nrf2 activity. Keap1 is found as a homodimer associated with Cullin-3, which binds to the BTB (Bric a brac) domain of Keap1, and is anchored to the actin cytoskeleton (Wakabayashi et al. 2004). In addition, some proteins are able to bind to the free site at Keap1, like the p62 protein (Jiang et al. 2015), thereby competing with Nrf2 for this binding site. Because of such binding, the closed conformational state cannot be formed. Altered expression of proteins that interact with the Keap1/Nrf2 complex during the first treatment may have consequences for the complex activity during a second treatment phase. Further work is required to identify the priming factors that drive an enhanced secondary anti-oxidant response and whether this response would occur for other bona fide Nrf2 target genes as well.

We set out to improve our fundamental understanding of cellular responses to repeat dosing scenarios. Altogether, our results demonstrate that cells previously exposed to pro-oxidants exhibit an altered response pattern compared to 'naive' cells. Importantly, such responses are also observed for drug molecules that are currently used in the clinic and show a severe-DILI liability (nitrofurantoin) or are in clinical trials (CDDO-Me). This involves both suppression of the activity of the transcription factor Nrf2 and priming for an enhanced upregulation of anti-oxidant molecules. Our findings could imply that a 'memory' mechanism is in place within the Nrf2 pathway in which cells previously exposed to xenobiotics are better protected against similar future exposures. These results have implications for the comprehension and translation of stress response activation for chemical safety assessment in daily life and drug treatment situations which typically involve repeat dose exposure scenarios.

Acknowledgements This work was supported by the Ministry of Defence of the Netherlands and the European Commission Horizon2020 EU-ToxRisk project (Grant nr 681002).

Open Access This article is distributed under the terms of the Creative Commons Attribution 4.0 International License (http://creativeco mmons.org/licenses/by/4.0/), which permits unrestricted use, distribution, and reproduction in any medium, provided you give appropriate credit to the original author(s) and the source, provide a link to the Creative Commons license, and indicate if changes were made.

\section{References}

Alumkal JJ, Slottke R, Schwartzman J et al (2015) A phase II study of sulforaphane-rich broccoli sprout extracts in men with recurrent prostate cancer. Invest New Drugs 33(2):480-489. https://doi. org/10.1007/s10637-014-0189-z

Baek JY, Han SH, Sung SH et al (2012) Sulfiredoxin protein is critical for redox balance and survival of cells exposed to low steadystate levels of H2O2. J Biol Chem 287(1):81-89. https://doi. org/10.1074/jbc.M111.316711

Baird L, Dinkova-Kostova AT (2011) The cytoprotective role of the Keap1-Nrf2 pathway. Arch Toxicol 85(4):241-272. https://doi. org/10.1007/s00204-011-0674-5

Baird L, Dinkova-Kostova AT (2013) Diffusion dynamics of the Keap1-Cullin3 interaction in single live cells. Biochem Biophys Res Commun 433(1):58-65. https://doi.org/10.1016/j. bbrc.2013.02.065

Bergstrom P, Andersson HC, Gao Y et al (2011) Repeated transient sulforaphane stimulation in astrocytes leads to prolonged Nrf2mediated gene expression and protection from superoxide-induced damage. Neuropharmacology 60(2-3):343-353. https://doi. org/10.1016/j.neuropharm.2010.09.023

Bryan HK, Olayanju A, Goldring CE, Park BK (2013) The Nrf2 cell defence pathway: Keap1-dependent and -independent mechanisms of regulation. Biochem Pharmacol 85(6):705-717. https://doi. org/10.1016/j.bcp.2012.11.016 
Casey W, Anderson S, Fox T, Dold K, Colton H, Morgan K (2002) Transcriptional and physiological responses of HepG2 cells exposed to diethyl maleate: time course analysis. Physiol Genomics 8(2):115-122. https://doi.org/10.1152/physiolgenomics.00064 .2001

Chang TS, Jeong W, Woo HA, Lee SM, Park S, Rhee SG (2004) Characterization of mammalian sulfiredoxin and its reactivation of hyperoxidized peroxiredoxin through reduction of cysteine sulfinic acid in the active site to cysteine. J Biol Chem 279(49):5099451001. https://doi.org/10.1074/jbc.M409482200

Chen W, Sun Z, Wang XJ et al (2009) Direct interaction between Nrf2 and $\mathrm{p} 21$ (Cip1/WAF1) upregulates the Nrf2-mediated antioxidant response. Mol Cell 34(6):663-673. https://doi.org/10.1016/j. molcel.2009.04.029

Cleasby A, Yon J, Day PJ et al (2014) Structure of the BTB domain of Keap1 and its interaction with the triterpenoid antagonist CDDO. PLoS One 9(6):e98896. https://doi.org/10.1371/journ al.pone. 0098896

Danial NN, Korsmeyer SJ (2004) Cell death: critical control points. Cell 116(2):205-219

Deshmukh P, Unni S, Krishnappa G, Padmanabhan B (2017) The Keap1-Nrf2 pathway: promising therapeutic target to counteract ROS-mediated damage in cancers and neurodegenerative diseases. Biophys Rev 9(1):41-56. https://doi.org/10.1007/s1255 1-016-0244-4

Fulda S, Gorman AM, Hori O, Samali A (2010) Cellular stress responses: cell survival and cell death. Int J Cell Biol 2010:214074. https://doi.org/10.1155/2010/214074

Herpers B, Wink S, Fredriksson L et al (2016) Activation of the Nrf2 response by intrinsic hepatotoxic drugs correlates with suppression of NF- $\mathrm{KB}$ activation and sensitizes toward TNF $\alpha-$ induced cytotoxicity. Arch Toxicol 90:1163-1179. https://doi. org/10.1007/s00204-015-1536-3

Hiemstra S, Niemeijer M, Koedoot E et al (2017) Comprehensive landscape of Nrf2 and p53 pathway activation dynamics by oxidative stress and DNA damage. Chem Res Toxicol 30(4):923933. https://doi.org/10.1021/acs.chemrestox.6b00322

Holland R, Fishbein JC (2010) Chemistry of the cysteine sensors in kelch-like ECH-associated protein 1. Antioxid Redox Signal 13(11):1749-1761. https://doi.org/10.1089/ars.2010.3273

Huang HC, Nguyen T, Pickett CB (2000) Regulation of the antioxidant response element by protein kinase C-mediated phosphorylation of NF-E2-related factor 2. Proc Natl Acad Sci USA 97(23):12475-12480. https://doi.org/10.1073/pnas.220418997

Imhoff BR, Hansen JM (2010) Tert-butylhydroquinone induces mitochondrial oxidative stress causing Nrf2 activation. Cell Biol Toxicol 26(6):541-551. https://doi.org/10.1007/s1056 5-010-9162-6

Itoh K, Chiba T, Takahashi S et al (1997) An Nrf2/small Maf heterodimer mediates the induction of phase II detoxifying enzyme genes through antioxidant response elements. Biochem Biophys Res Commun 236(2):313-322

Jennings P, Limonciel A, Felice L, Leonard MO (2013) An overview of transcriptional regulation in response to toxicological insult. Arch Toxicol 87(1):49-72. https://doi.org/10.1007/s00204-012-0919-y

Jiang T, Harder B, Rojo de la Vega M, Wong PK, Chapman E, Zhang DD (2015) p62 links autophagy and Nrf2 signaling. Free Radic Biol Med 88(Pt B):199-204. https://doi.org/10.1016/j.freeradbio med.2015.06.014

Kamentsky L, Jones TR, Fraser A et al (2011) Improved structure, function and compatibility for CellProfiler: modular high-throughput image analysis software. Bioinformatics 27(8):1179-1180. https://doi.org/10.1093/bioinformatics/btr095

Katsuoka F, Motohashi H, Engel JD, Yamamoto M (2005) Nrf2 transcriptionally activates the mafG gene through an antioxidant response element. J Biol Chem 280(6):4483-4490. https://doi. org/10.1074/jbc.M411451200

Kensler TW, Wakabayashi N, Biswal S (2007) Cell survival responses to environmental stresses via the Keap1-Nrf2-ARE pathway. Annu Rev Pharmacol Toxicol 47:89-116. https://doi.org/10.1146/annur ev.pharmtox.46.120604.141046

Keum YS, Choi BY (2014) Molecular and chemical regulation of the Keap1-Nrf2 signaling pathway. Molecules 19(7):10074-10089. https://doi.org/10.3390/molecules190710074

Keum YS, Han YH, Liew C et al (2006) Induction of heme oxygenase-1 (HO-1) and NAD[P]H: quinone oxidoreductase 1 (NQO1) by a phenolic antioxidant, butylated hydroxyanisole (BHA) and its metabolite, tert-butylhydroquinone (tBHQ) in primary-cultured human and rat hepatocytes. Pharm Res 23(11):2586-2594. https ://doi.org/10.1007/s11095-006-9094-2

Kobayashi A, Kang MI, Okawa H et al (2004) Oxidative stress sensor Keap1 functions as an adaptor for Cul3-based E3 ligase to regulate proteasomal degradation of Nrf2. Mol Cell Biol 24(16):71307139. https://doi.org/10.1128/MCB.24.16.7130-7139.2004

Kobayashi A, Kang MI, Watai Y et al (2006) Oxidative and electrophilic stresses activate Nrf2 through inhibition of ubiquitination activity of Keap1. Mol Cell Biol 26(1):221-229. https://doi. org/10.1128/MCB.26.1.221-229.2006

Kobayashi M, Li L, Iwamoto $\mathrm{N}$ et al (2009) The antioxidant defense system Keap1-Nrf2 comprises a multiple sensing mechanism for responding to a wide range of chemical compounds. Mol Cell Biol 29(2):493-502. https://doi.org/10.1128/MCB.01080-08

Lee JM, Moehlenkamp JD, Hanson JM, Johnson JA (2001) Nrf2dependent activation of the antioxidant responsive element by tert-butylhydroquinone is independent of oxidative stress in IMR32 human neuroblastoma cells. Biochem Biophys Res Commun 280(1):286-292. https://doi.org/10.1006/bbrc.2000.4106

Lynch R, Diggins EL, Connors SL et al (2017) Sulforaphane from broccoli reduces symptoms of autism: a follow-up case series from a randomized double-blind study. Glob Adv Health Med 6:2164957X17735826. https://doi.org/10.1177/2164957X17 735826

Ma Q (2013) Role of nrf2 in oxidative stress and toxicity. Annu Rev Pharmacol Toxicol 53:401-426. https://doi.org/10.1146/annurevpharmtox-011112-140320

Mathew ST, Bergstrom P, Hammarsten O (2014) Repeated Nrf2 stimulation using sulforaphane protects fibroblasts from ionizing radiation. Toxicol Appl Pharmacol 276(3):188-194. https://doi. org/10.1016/j.taap.2014.02.013

Meakin PJ, Chowdhry S, Sharma RS et al (2014) Susceptibility of Nrf2-null mice to steatohepatitis and cirrhosis upon consumption of a high-fat diet is associated with oxidative stress, perturbation of the unfolded protein response, and disturbance in the expression of metabolic enzymes but not with insulin resistance. Mol Cell Biol 34(17):3305-3320. https://doi.org/10.1128/MCB.00677 $-14$

Poser I, Sarov M, Hutchins JR et al (2008) BAC TransgeneOmics: a high-throughput method for exploration of protein function in mammals. Nat Methods 5(5):409-415. https://doi.org/10.1038/ nmeth.1199

Priya S, Nigam A, Bajpai P, Kumar S (2014) Diethyl maleate inhibits MCA + TPA transformed cell growth via modulation of GSH, MAPK, and cancer pathways. Chem Biol Interact 219:37-47. https://doi.org/10.1016/j.cbi.2014.04.018

Ryter SW, Kim HP, Hoetzel A et al (2007) Mechanisms of cell death in oxidative stress. Antioxid Redox Sign 9(1):49-89. https://doi. org/10.1089/ars.2007.9.49

Soriano FX, Leveille F, Papadia S et al (2008) Induction of sulfiredoxin expression and reduction of peroxiredoxin hyperoxidation by the neuroprotective $\mathrm{Nrf} 2$ activator $3 \mathrm{H}$-1,2-dithiole-3-thione. 
J Neurochem 107(2):533-543. https://doi.org/10.111 1/j.1471-4159.2008.05648.x

Souza TM, Kleinjans JCS, Jennen DGJ (2017) Dose and time dependencies in stress pathway responses during chemical exposure: novel insights from gene regulatory networks. Front Genet 8:142. https://doi.org/10.3389/fgene.2017.00142

Sun Z, Chin YE, Zhang DD (2009) Acetylation of Nrf2 by p300/CBP augments promoter-specific DNA binding of Nrf2 during the antioxidant response. Mol Cell Biol 29(10):2658-2672. https://doi. org/10.1128/mcb.01639-08

Tsuchiya T, Kijima A, Ishii Y et al (2018) Role of oxidative stress in the chemical structure-related genotoxicity of nitrofurantoin in Nrf2-deficient gpt delta mice. J Toxicol Pathol 31(3):169-178. https://doi.org/10.1293/tox.2018-0014

Vomund S, Schafer A, Parnham MJ, Brune B, von Knethen A (2017) $\mathrm{Nrf2}$, the master regulator of anti-oxidative responses. Int J Mol Sci 18(12) https://doi.org/10.3390/ijms 18122772

Wakabayashi N, Dinkova-Kostova AT, Holtzclaw WD et al (2004) Protection against electrophile and oxidant stress by induction of the phase 2 response: fate of cysteines of the Keap1 sensor modified by inducers. Proc Natl Acad Sci USA 101(7):2040-2045. https:// doi.org/10.1073/pnas.0307301101

Wang X-Y, Zhang X-H, Peng L et al (2017) Bardoxolone methyl (CDDO-Me or RTA402) induces cell cycle arrest, apoptosis and autophagy via PI3K/Akt/mTOR and p38 MAPK/Erk1/2 signaling pathways in K562 cells. Am J Transl Res 9(10):4652-4672

Wink S, Hiemstra S, Herpers B, van de Water B (2017) High-content imaging-based BAC-GFP toxicity pathway reporters to assess chemical adversity liabilities. Arch Toxicol 91(3):1367-1383. https://doi.org/10.1007/s00204-016-1781-0

Wink S, Hiemstra SW, Huppelschoten S, Klip JE, van de Water B (2018) Dynamic imaging of adaptive stress response pathway activation for prediction of drug induced liver injury. Arch Toxicol. https://doi.org/10.1007/s00204-018-2178-z

Wu KC, Cui JY, Klaassen CD (2012) Effect of graded Nrf2 activation on phase-I and -II drug metabolizing enzymes and transporters in mouse liver. PLoS One 7(7):e39006. https://doi.org/10.1371/ journal.pone.0039006

Yamauchi S, Kiyosawa N, Ando Y et al (2011) Hepatic transcriptome and proteome responses against diethyl maleate-induced glutathione depletion in the rat. Arch Toxicol 85(9):1045-1056. https ://doi.org/10.1007/s00204-010-0632-7

Yan K, Verbeek FJ (2012) Segmentation for high-throughput image analysis: watershed masked clustering. In: Berlin, Heidelberg, 2012. Leveraging applications of formal methods, verification and validation. Applications and case studies. Springer, Berlin, Heidelberg, pp 25-41

Yeakley JM, Shepard PJ, Goyena DE, VanSteenhouse HC, McComb JD, Seligmann BE (2017) A trichostatin A expression signature identified by TempO-Seq targeted whole transcriptome profiling. PLoS One 12(5):e0178302-e0178302. https://doi.org/10.1371/ journal.pone. 0178302

Zhang DD, Lo SC, Cross JV, Templeton DJ, Hannink M (2004) Keap1 is a redox-regulated substrate adaptor protein for a Cul3-dependent ubiquitin ligase complex. Mol Cell Biol 24(24):10941-10953. https://doi.org/10.1128/MCB.24.24.10941-10953.2004

Zhou Y, Duan S, Zhou Y et al (2015) Sulfiredoxin-1 attenuates oxidative stress via Nrf2/ARE pathway and 2-Cys Prdxs after oxygenglucose deprivation in astrocytes. J Mol Neurosci 55(4):941-950. https://doi.org/10.1007/s12031-014-0449-6

Zipper LM, Mulcahy RT (2002) The Keap1 BTB/POZ dimerization function is required to sequester Nrf2 in cytoplasm. J Biol Chem 277(39):36544-36552. https://doi.org/10.1074/jbc.M206530200 